\title{
LIMITING DYNAMICS FOR SPHERICAL MODELS OF SPIN GLASSES AT HIGH TEMPERATURE
}

\author{
AMIR DEMBO, ALICE GUIONNET, AND CHRISTIAN MAZZA
}

\begin{abstract}
We analyze the coupled non-linear integro-differential equations whose solution is the thermodynamical limit of the empirical correlation and response functions in the Langevin dynamics for spherical $p$-spin disordered mean-field models. We provide a mathematically rigorous derivation of their FDT solution (for the high temperature regime) and of certain key properties of this solution, which are in agreement with earlier derivations based on physical grounds.
\end{abstract}

\section{INTRODUCTION}

The complex long time behavior predicted for the thermodynamical limits of a wide class of Markovian dynamics with random interactions, is among the fascinating aspects of out of equilibrium statistical physics (for a good survey on phenomena such as aging, memory, rejuvenation, and violation of the FluctuationDissipation Theorem (FDT), see $[7,8]$ ). This work is concerned with the long time behavior of a complex system composed of $N$ Langevin particles $x_{t}=\left(x_{t}^{i}\right)_{1 \leq i \leq N} \in \mathbb{R}^{N}$, each evolving in $\mathbb{R}$ and interacting with the others through a random potential. More precisely, one considers a diffusion of the form

$$
d x_{t}=-f^{\prime}\left(\left\|x_{t}\right\|^{2} / N\right) x_{t} d t-\beta \nabla H_{J}\left(x_{t}\right) d t+d B_{t},
$$

where $B_{t}$ is a $N$-dimensional Brownian motion, $\|x\|$ denotes the Euclidean norm of $x \in \mathbb{R}^{N}$ and $f$ is a convex function. Such models are called spherical (for well chosen $f, x_{t}$ is restricted to stay on a sphere). The mixed $p$-spin, $p \leq m$, potential $H_{J}: \mathbb{R}^{N} \longrightarrow \mathbb{R}$ is given by

$$
H_{J}(x)=\sum_{p=2}^{m} \frac{a_{p}}{p !} \sum_{1 \leq i_{1} \leq \cdots \leq i_{p} \leq N} J_{i_{1} \cdots i_{p}} x^{i_{1}} \cdots x^{i_{p}}, \quad a_{m} \neq 0
$$

where the coupling constants $J_{i_{1} \cdots i_{p}}$ are assumed to be independent centered Gaussian variables. The variance of $J_{i_{1} \ldots i_{p}}$ is $c\left(\left\{i_{1}, \ldots, i_{p}\right\}\right) N^{-p+1}$, where

$$
c\left(\left\{i_{1}, \ldots, i_{p}\right\}\right)=\prod_{k} l_{k} !,
$$

and $\left(l_{1}, l_{2}, \ldots\right)$ are the multiplicities of the different elements of the set $\left\{i_{1}, \ldots, i_{p}\right\}$ (for example, $c=1$ when $i_{j} \neq i_{j^{\prime}}$ for any $j \neq j^{\prime}$, while $c=p$ ! when all $i_{j}$ values are the same).

When $m=2$, one gets the so-called Sherrington-Kirckpatrick spherical spin glass, which has been studied in details in [3]. Given a realization of the coupling constants, the dynamics of (1.1) is invariant for the (random) Gibbs measure

$$
\mu_{N}^{J}(d x)=Z_{J, N}^{-1} \exp \left(-N f\left(\|x\|^{2} / N\right)-2 \beta H_{J}(x)\right) \prod_{i=1}^{N} d x^{i}
$$

Research partially supported by NSF grants \#DMS-0406042, \#DMS-FRG-0244323. Supported in parts by the Swiss National Foundation.

AMS (2000) Subject Classification: Primary: 82C44 Secondary: 82C31, 60H10, 60F15, 60K35

Keywords: Interacting random processes, Disordered systems, Statistical mechanics, Langevin dynamics, Aging, $p$-spin models. 
Similar random measures have been extensively studied in mathematics and physics during the last two decades (see e.g. [20], for the rigorous analysis of the asymptotics of the free energy of the measure with a hard spherical constraint, corresponding to spins on the sphere $\left.\|x\|^{2}=N\right)$. Here, we shall be concerned with the statistical properties of the dynamics at high temperature. The natural quantity of interest is the empirical covariance function

$$
C_{N}(s, t)=\frac{1}{N} \sum_{i=1}^{N} x_{s}^{i} x_{t}^{i}, \quad s \geq t,
$$

in the large $N$ limit, and for large $t$ and $s$. It turns out that the asymptotic behavior of (1.5) strongly depends on the way $t$ and $s$ tend to infinity, at least at low temperature. This is a trace of aging: the older it gets, the longer the system will take to forget its age. This innocent looking notion of aging is related to deep mathematical problems (see e.g. the survey in [13]), and leads to interesting mathematical scenarios, like the ultrametric property of the covariance function at low temperature (see e.g. [10]). In [3], the authors present a detailed analysis of the aging properties of (1.5) in the special case $m=2$, using integro differential equations involving the almost sure limit $C(s, t)=\lim _{N \rightarrow \infty} C_{N}(s, t)$. When $m \neq 2$, closed equations for $C$ are obtained in [10] (for the hard spherical constraint) and rigorously derived in [5], where they are called Cugliandolo-Kurchan equations. These equations involve also the limit of the integrated response function

$$
\chi_{N}(s, t)=\frac{1}{N} \sum_{i=1}^{N} x_{s}^{i} B_{t}^{i} .
$$

According to [5], fixing $T<\infty$, the random functions $C_{N}$ and $\chi_{N}$ converge uniformly on $[0, T]^{2}$, almost surely and in $L_{1}$ to non-random functions $C(s, t)$ and $\chi(s, t)=\int_{0}^{t} R(s, u) d u$ with $R(s, t)=0$ when $t>s, R(s, s) \equiv 1$, and, for $s>t$, the absolutely continuous functions $C, R$ and $K(s)=C(s, s)$ are the unique solutions in the space of bounded, continuous functions, of the non linear integro-differential equations

$$
\begin{aligned}
\partial_{s} R(s, t) & =-f^{\prime}(K(s)) R(s, t)+\beta^{2} \int_{t}^{s} R(u, t) R(s, u) \nu^{\prime \prime}(C(s, u)) d u \\
\partial_{s} C(s, t) & =-f^{\prime}(K(s)) C(s, t)+\beta^{2} \int_{0}^{s} C(u, t) R(s, u) \nu^{\prime \prime}(C(s, u)) d u+\beta^{2} \int_{0}^{t} \nu^{\prime}(C(s, u)) R(t, u) d u \\
\partial_{s} K(s) & =-2 f^{\prime}(K(s)) K(s)+1+2 \beta^{2} \int_{0}^{s} \psi(C(s, u)) R(s, u) d u
\end{aligned}
$$

where $\psi(r)=\nu^{\prime}(r)+r \nu^{\prime \prime}(r)$,

$$
\nu(r)=\sum_{p=2}^{m} \frac{a_{p}^{2}}{p !} r^{p}, \quad m \geq 2, \quad a_{m} \neq 0,
$$

and the initial condition $K(0)=C(0,0)>0$ is given. It was shown in Theorem 1.2 of [5] that for

$$
f(r):=f_{L}(r)=L(r-1)^{2}+\frac{1}{4 k} r^{2 k}, \quad k>m / 4, k \in \mathbb{Z}, L \geq 0,
$$

these equations admit a unique solution $C(s, t)=C(t, s), R(s, t)$ and $K(s)=C(s, s)$ in the space of absolutely continuous functions on $\{(s, t): 0 \leq t \leq s\}$. $^{1}$

Whereas [5] rigorously derives these equations using stochastic calculus and concentration inequalities, in the physics literature they are attained via the so-called Martin-Siggia-Rose formalism (see e.g. [6] or [9]). An alternative to the latter is to expand the stochastic process $x_{t}$ perturbatively using diagrams, as explained for example in [6] and [8], then average over the disorder the product $x_{s} x_{t}$ to get equations relating the covariance and response functions. The generic form of the family of diagrams one uses in this process

\footnotetext{
${ }^{1}$ More general choice of differentiable $f(\cdot)$ is allowed in [5], but we focus here on the collection given by (1.11).
} 
indicates that (1.7)-(1.9) are equivalent to a class of mode coupling equations (see e.g. [6], [8] or [17]). Mode coupling approximations were developed in physics to study nonlinear random dynamical systems occurring in many contexts like plasma physics, kinetic theory of classical liquids or glasses (see e.g. [15] and the references therein). This method considers a perturbative expansion as a series indexed by diagrams containing information on the nonlinearity. The series is then renormalized, to produce self-consistent moment equations, called mode coupling equations. Kraichnan [16] [17] developed such approximations (called direct interaction in fluid mechanics) when considering the solutions to the Navier-Stokes equations in Fourier space under random initial conditions, and gave a procedure to perform the statistical closure of the moment equations: usually, there is a cascade of moments meaning that the time derivative of the second moment involve the third moment and so on. One then looks for good approximations leading to self-consistent equations (c.f. the texts [18] and [19], for a variety of statistical closure problems and diagrammatic methods in fluid mechanics). More recently, these approximations were applied in the study of super-cooled and strongly interacting liquids (glasses), producing very accurate quantitative predictions (see [14], [15]). The fundamental object of interest in structural glasses is a correlation function $\eta(t)$, for which the mode coupling equation is

$$
\eta^{\prime \prime}(t)+\Omega^{2} \eta(t)+\nu \eta^{\prime}(t)+\Omega^{2} \int_{0}^{t} k(\eta(t-u)) \eta^{\prime}(u) \mathrm{d} u=0,
$$

where $k(r)=\sum_{p=1}^{m} b_{p} r^{p}$, for some constants $\Omega, \nu$ and $\left\{b_{p}, p=1, \ldots, m\right\}$ (see e.g. [14]). The integro-differential equation (1.12) is similar to what one gets when postulating the FDT ansatz, whereby the solution $(R, C)$ of (1.7)-(1.9) is translation invariant, with $K(s)$ constant and $R$ proportional to the derivative of $C$ (for example, see (5.9) in the sequel). Of course, the first task when dealing with (1.7)-(1.9) is to show the validity of this ansatz, at least for $\beta$ small enough, as we do in Theorem 1.3. Further, the genericity of (1.12) implies that any relevant information about its solution is of interest (and in this context see Proposition 1.4).

The asymptotic behavior of $C(s, t)$ and $R(s, t)$ for large values of $t$ and $s$ is difficult to pin down; in [10], the authors propose various scenarios, but no complete description of these asymptotics could be given (see also [7] or [8]). A first regime of interest is the so-called FDT regime, in which the fluctuation dissipation theorem of statistical physics is expected to hold. In this regime, the covariance should be stationary, that is, for fixed $s-t=\tau$ and $t$ large, the covariance $C(s, t)$ should be approximated well by some function $C_{\mathrm{fdt}}(\tau)$. Further, from [12] we know that the response function $R(s, t)$ is then well approximated by some function $R_{\mathrm{fdt}}(\tau)$. In this regime we further expect the FDT relation $R_{\mathrm{fdt}}(\tau)=-2 C_{\mathrm{fdt}}^{\prime}(\tau)$ to hold (the proportionality constant has to do with the scaling we employed in the definition of $R(s, t)$ and not with its physical meaning). We shall see in this work that the FDT regime holds for small enough $\beta$ in which case both $C_{\mathrm{fdt}}$ and $R_{\mathrm{fdt}}$ decay to zero exponentially fast. The aging regime is expected to be characterized by covariances and response functions of the generic form $C(s, t)=C_{\text {aging }}(h(t) / h(s))$ and $R(s, t)=h^{\prime}(t) h(s)^{-1} R_{\text {aging }}(h(t) / h(s))$ with sub-exponential growth of the monotone function $h(\cdot)$ and a polynomial decay to zero and to appear only for $\beta>\beta_{c}$, the dynamical phase transition point of (1.1). These different scenarios are examined in [12], where fixing the asymptotic behavior of $C$ according to the above choices $C_{\mathrm{fdt}}$ or $C_{\text {aging }}$, the authors study the solution $\widetilde{R}$ of the equation

$$
\partial_{s} \widetilde{R}(s, t)=-f^{\prime}(K(s)) \widetilde{R}(s, t)+\int_{t}^{s} \widetilde{R}(u, t) \widetilde{R}(s, u) k(s, u) d u,
$$

where $k(s, u)$ stands either for $\beta^{2} \nu^{\prime \prime}\left(C_{\mathrm{fdt}}(s-u)\right)$ or for $\beta^{2} \nu^{\prime \prime}\left(C_{\text {aging }}(h(u) / h(s))\right.$. Let

$$
H(s, t)=\exp \left(\int_{t}^{s} f^{\prime}(K(u)) d u\right) R(s, t) .
$$

Then it is easy to check that $H$ solves the equation

$$
\partial_{s} H(s, t)=\beta^{2} \int_{t}^{s} H(s, u) H(u, t) \nu^{\prime \prime}(C(s, u)) d u, \quad H(t, t)=1 .
$$


The main technique of [12] is a formal solution to (1.13) involving non-crossing involutions. We shall also use this formula in the sequel to prove various statements concerning the solution $C$ and $R$ of the complete system of equations (for example, their exponential decay at high enough temperature).

We first show that in the limit $L \rightarrow \infty$, the solutions of the equations (1.7)-(1.9) for our "soft" spherical constraint by the potential $f_{L}(\cdot)$ coincide with the limiting equations of [10] for the hard spherical constraint (where $C(2 \cdot, 2 \cdot)$ and $R(2 \cdot, 2 \cdot)$ are considered for $\nu(r)=r^{p} / 8$ ).

Proposition 1.1. For any $T<\infty$, the solution $\left(R_{L}, K_{L}, C_{L}\right)$ of the system (1.7)-(1.9) with potential $f_{L}(\cdot)$ as in (1.11) and initial condition $K_{L}(0)=1$, converges as $L \rightarrow \infty$, uniformly in $[0, T]^{2}$, towards the triplet $(R, K, C)$ such that $C(t, t)=K(t)=1$ for all $t \geq 0, R(s, t)=0$ for all $s<t$, and for all $s \geq t$,

$$
\begin{aligned}
& \partial_{s} R(s, t)=-\mu(s) R(s, t)+\beta^{2} \int_{t}^{s} R(u, t) R(s, u) \nu^{\prime \prime}(C(s, u)) d u, \\
& \partial_{s} C(s, t)=-\mu(s) C(s, t)+\beta^{2} \int_{0}^{s} C(u, t) R(s, u) \nu^{\prime \prime}(C(s, u)) d u+\beta^{2} \int_{0}^{t} \nu^{\prime}(C(s, u)) R(t, u) d u,
\end{aligned}
$$

where

$$
\mu(s)=\frac{1}{2}\left(1+2 \beta^{2} \int_{0}^{s} \psi(C(s, u)) R(s, u) d u\right) .
$$

Moreover, $C(s, t)=C(t, s)$ is a non-negative definite kernel, with values in $[0,1]$ and $R(s, t) \geq 0$ is such that $R(t, t)=1$ and

$$
\left|\int_{t_{1}}^{t_{2}} R(s, u) d u\right|^{2} \leq t_{2}-t_{1}, \quad 0 \leq t_{1} \leq t_{2} \leq s<\infty .
$$

We prove in Section 3 that for $\beta>0$ sufficiently small, the solution $(R, C)$ of $(1.14)-(1.16)$ decays exponentially fast in $s-t$.

Proposition 1.2. There exists $\beta_{0}>0$ such that for all $\beta<\beta_{0}$ there exists $M_{\beta}<\infty$ and $\eta_{\beta}>0$ for which the solution of (1.14)-(1.16) satisfies for all $s \geq t \geq 0$,

$$
\begin{aligned}
& R(s, t) \leq e^{-\eta_{\beta}(s-t)} \\
& C(s, t) \leq M_{\beta} e^{-\eta_{\beta}(s-t)} .
\end{aligned}
$$

Equipped with Proposition 1.2 we prove in Section 4 that for some $\beta_{1}>0$ and each $\beta<\beta_{1}$, when $s-t=\tau$ is fixed and $t \rightarrow \infty$ the solution $(R(s, t), C(s, t))$ converges to a limiting pair $\left(R_{\mathrm{fdt}}(\tau), C_{\mathrm{fdt}}(\tau)\right)$ that satisfies the FDT relation $R_{\mathrm{fdt}}(\tau)=-2 C_{\mathrm{fdt}}^{\prime}(\tau)$ for all $\tau \geq 0$. Upon analyzing in Section 5 the corresponding FDT equations (4.15)-(4.17), we establish our main result.

Theorem 1.3. If $\beta<\beta_{1} \leq 1 /\left(2 \sqrt{\nu^{\prime \prime}(0)}\right)$ then for any $\tau \geq 0$,

$$
\lim _{t \rightarrow \infty} C(\tau+t, t)=C_{\mathrm{fdt}}(\tau)
$$

and

$$
\lim _{t \rightarrow \infty} R(\tau+t, t)=R_{\mathrm{fdt}}(\tau)=-2 C_{\mathrm{fdt}}^{\prime}(\tau),
$$

where $C_{\mathrm{fdt}}(\cdot)$ is the unique $[0,1]$-valued, continuously differentiable solution of the equation

$$
D^{\prime}(s)=-\int_{0}^{s} \phi(D(v)) D^{\prime}(s-v) d v-b, \quad D(0)=1,
$$

for $b=1 / 2$ and $\phi(x)=b+2 \beta^{2} \nu^{\prime}(x)$. Moreover, both $C_{\mathrm{fdt}}(\cdot)$ and $R_{\mathrm{fdt}}(\cdot)$ decay exponentially to zero at infinity.

A key ingredient of the proof of Theorem 1.3 is the analysis of the equation (1.20), which is of some independent interest. Specifically, in Section 5 we prove the following. 
Proposition 1.4. Suppose $b>0$ and $\phi:[0,1] \rightarrow \mathbb{R}$ is non-decreasing, of Lipschitz continuous derivative such that

$$
\sup _{0 \leq x \leq 1}\{\phi(x)(1-x)\} \geq b .
$$

Then, (1.20) has a unique solution in the space of [0,1]-valued continuously differentiable functions. This solution is twice continuously differentiable, strictly decreasing and converges for $s \rightarrow \infty$ to

$$
D_{\infty}:=\sup \{x \in[0,1]: \phi(x)(1-x) \geq b\} .
$$

Further, in case $\phi(\cdot)$ is convex and $\phi(1)>2 \sqrt{b \phi^{\prime}(1)}$, the derivative of the solution of (1.20) decays exponentially to zero with some positive exponent.

Setting $b=\phi\left(D_{\infty}\right)\left(1-D_{\infty}\right)$ and considering $x \downarrow D_{\infty}$ in $(1.21)$, it is not hard to verify that necessarily $\phi\left(D_{\infty}\right) \geq \phi^{\prime}\left(D_{\infty}\right)\left(1-D_{\infty}\right)$. In the remark following the proof of Proposition 1.4 we observe that the condition

$$
\phi\left(D_{\infty}\right)>\phi^{\prime}\left(D_{\infty}\right)\left(1-D_{\infty}\right)
$$

is necessary for the exponential convergence of $D^{\prime}(s)$ to zero as $s \rightarrow \infty$ when $\phi(\cdot)$ is convex. As (1.22) is easily seen to be equivalent to $\phi(1)>2 \sqrt{b \phi^{\prime}(1)}$ when $\phi(x)$ is linear, we speculate that it actually characterizes the exponential convergence of the solution of (1.20).

Of course, in case of $\phi(\cdot)=b+2 \beta^{2} \nu^{\prime}(\cdot)$ and $b=1 / 2$ the condition (1.21) holds (try $x=0$ ). Setting $\beta_{c} \in(0, \infty)$ via

$$
\frac{1}{4 \beta_{c}^{2}}=\sup \left\{\nu^{\prime}(x)(1-x) x^{-1}: x \in(0,1]\right\}
$$

it is easy to check that in this case $D_{\infty}=0$ if $\beta<\beta_{c}$ whereas $D_{\infty}>0$ for $\beta>\beta_{c}$. Further, considering $x \rightarrow 0$ in (1.23) we find that $1 /\left(4 \beta_{c}^{2}\right) \geq \nu^{\prime \prime}(0)$, so the condition (1.22) then holds for any $\beta<\beta_{c}$. This indicates that though the values of $\beta_{0}$ and $\beta_{1}$ in our proofs of Proposition 1.2 and Theorem 1.3 are quite small, both should match the predicted dynamical phase transition point $\beta_{c}$ of our model.

Indeed, subject to a heuristic ansatz, similar to what can be found in the physics literature, we show in Section 6 why the equation (1.20) with $b=1 / 2$ and $\phi(x)=\gamma+2 \beta^{2} \nu^{\prime}(x)$ describes the FDT regime at all temperatures, albeit in general with $\gamma=\gamma(\beta) \neq b$ (see Proposition 6.1). More precisely, the physics prediction for the choice of $\gamma$ is as follows. Let $x^{*}$ denote the largest value of $x<1$ attaining the supremum in (1.23) of the polynomial $h(x)=\nu^{\prime}(x)(1-x) / x$ (with $h(0)=\nu^{\prime \prime}(0)$ ). Setting $g(x)=\nu^{\prime \prime}(x)(1-x)^{2}$ we have that $g\left(x^{*}\right)=h\left(x^{*}\right)=1 /\left(4 \beta_{c}^{2}\right)$ as a consequence of the optimality condition $h^{\prime}\left(x^{*}\right)=0$ if $x^{*}>0$ (while trivially $g(0)=h(0))$. From the continuity of $g(\cdot)$ and the fact that $g(1)=0$ it follows that

$$
q(\beta):=\sup \left\{x \in[0,1]: 4 \beta^{2} \nu^{\prime \prime}(x)(1-x)^{2} \geq 1\right\},
$$

is in $\left[x^{*}, 1\right)$ for all $\beta \geq \beta_{c}$, with $q(\beta)>x^{*}$ as soon as $\beta>\beta_{c}$. Setting $\gamma(\beta)=2 \beta^{2}\left[\nu^{\prime \prime}(x)(1-x)-\nu^{\prime}(x)\right]$ for $x=q(\beta)$ we find that the condition (1.21) of Proposition 1.4 applies also for $\beta \geq \beta_{c}$. Further, the expected limit $D_{\infty}$ of $C_{\mathrm{fdt}}(\tau)$ as $\tau \rightarrow \infty$ is the preceding $q(\beta)$ which is strictly positive for $\beta>\beta_{c}$, indicating the onset of the aging regime at $\beta_{c}$. Another indication of the onset of aging is the fact that when $\beta \geq \beta_{c}$ the exponential convergence of $C_{\mathrm{fdt}}(\cdot)$ and $R_{\mathrm{fdt}}(\cdot)$ is lost (i.e. the preceding choice of $\gamma(\beta)$ leads to equality in (1.22)). The physics prediction suggests also that

$$
\mathbf{I}_{\gamma(\beta)}=\beta^{2} \int_{0}^{1} \psi\left(C_{\text {aging }}(\lambda)\right) R_{\text {aging }}(\lambda) d \lambda \geq 0,
$$

where $\mathbf{I}_{\gamma(\beta)}=\gamma(\beta)-\frac{1}{2}+2 \beta^{2} q(\beta) \nu^{\prime}(q(\beta))$ is strictly positive when $q(\beta)>0$, and in particular, whenever $\beta>\beta_{c}$ (c.f. the remark following Proposition 6.1). The precise nature of the dynamical phase transition at $\beta_{c}$ depends on whether $q\left(\beta_{c}\right)$ is strictly positive (as is the case for example whenever $a_{2}=0$ ), or not. The physics ansatz of one aging regime with $R_{\text {aging }}(\lambda)=A C_{\text {aging }}^{\prime}(\lambda)$ and $C_{\text {aging }}(\cdot)$ monotone increasing on $[0,1]$ from $C_{\text {aging }}(0)=0$ to $C_{\text {aging }}(1)=q(\beta)$, proposed in [10] for the case in which $q\left(\beta_{c}\right)>0$, thus allows one to 
set the positive constant $A=\mathbf{I}_{\gamma(\beta)} /\left(\beta^{2} q(\beta) \nu^{\prime}(q(\beta))\right.$ in terms of the FDT solution. Unfortunately, even under this ansatz, the form of $C_{\text {aging }}(\cdot)$ is yet unclear.

Finally, note that it is crucial for our analysis to have $\nu^{\prime}(0)=0$, an assumption we make throughout this work. That is, our analysis holds in the absence of a random magnetic field.

\section{LIMITING EXACTLY SPHERICAL DYNAMICS}

Proposition 1.3 and (2.13) of [5] show that $\sup _{t \geq 0} K(t)<\infty$. Further, as $C(s, t)$ is the limit of the empirical correlation functions $C_{N}(s, t)$ it is a non-negative definite kernel on $\mathbb{R}_{+} \times \mathbb{R}_{+}$and in particular, $C(s, t)^{2} \leq K(s) K(t)$, whereas since $\chi(s, t)=\int_{0}^{t} R(s, u) d u$ is the limit of $\chi_{N}(s, t)$, it follows from the definition of $\chi_{N}(s, t)$ that

$$
\left|\int_{t_{1}}^{t_{2}} R(s, u) d u\right|^{2} \leq K(s)\left(t_{2}-t_{1}\right), \quad 0 \leq t_{1} \leq t_{2} \leq s<\infty
$$

To complete the proof of Proposition 1.1, we first prove that any solution $(R, C, K)$ of $(1.7)-(1.9)$ consists of positive functions, a key fact in our forthcoming analysis.

Lemma 2.1. For any $f: \mathbb{R}_{+} \rightarrow \mathbb{R}$ whose derivative is bounded above on compact intervals and any $K(0)>0$, a solution $(R, C, K)$ to $(1.7)-(1.9)$, if it exists, is positive at all times.

Proof: By definition $K(t) \geq 0$ for all $t \in \mathbb{R}_{+}$. Suppose that

$$
S=\inf \{u \geq 0: C(t, u) \leq 0 \text { for some } t \leq u\}<\infty .
$$

By continuity of $(C, K)$, since $K(0)>0$ also $S>0$. Set $\Lambda(s, t)=\exp \left(-\int_{t}^{s} \mu(u) d u\right)>0$ for $\mu(u)=f^{\prime}(K(u))$ which is bounded above on compact intervals, and $R(s, t)=\Lambda(s, t) H(s, t)$. Then, by [12], for $s \geq t$,

$$
H(s, t)=1+\sum_{n \geq 1} \beta^{2 n} \sum_{\sigma \in \mathrm{NC}_{n}} \int_{t \leq t_{1} \cdots \leq t_{2 n} \leq s} \prod_{i \in \operatorname{cr}(\sigma)} \nu^{\prime \prime}\left(C\left(t_{i}, t_{\sigma(i)}\right)\right) \prod_{j=1}^{2 n} d t_{j}
$$

where $\mathrm{NC}_{n}$ denotes the set of involutions of $\{1, \cdots, 2 n\}$ without fixed points and without crossings and $\operatorname{cr}(\sigma)$ is defined to be the set of indices $1 \leq i \leq 2 n$ such that $i<\sigma(i)$. Consequently,

$$
R(s, t) \geq \Lambda(s, t)>0 \text { for } t \leq s \leq S,
$$

and thus, (1.8) implies that also

$$
C(s, t) \geq K(t) \Lambda(s, t) \text { for } t \leq s \leq S .
$$

Note that in the last two estimates we used the fact that $\nu^{\prime}(\cdot)$ and $\nu^{\prime \prime}(\cdot)$ are non negative on $\mathbb{R}_{+}$. Similarly, from the equation (1.9) we see that $\partial_{s}\left[\Lambda(s, 0)^{-2} K(s)\right] \geq \Lambda(s, 0)^{-2}$ for all $s \leq S$ resulting with

$$
K(s) \geq K(0) \Lambda(s, 0)^{2}+\int_{0}^{s} \Lambda(s, v)^{2} d v>0
$$

Hence, the continuous functions $R(s, t), C(s, t)$ and $K(s)$ are bounded below by a strictly positive constant for $t \leq s \leq S$ in contradiction with the definition of $S$. We thus deduce that $S=\infty$ and by the preceding argument both $R(s, t)$ and $C(s, t)$ are positive functions.

We next show that if $\left(R_{L}, C_{L}, K_{L}\right)$ are solutions of the system (1.7)-(1.9) with potential $f_{L}(\cdot)$ as in (1.11), then $K_{L}(s) \rightarrow 1$ as $L \rightarrow \infty$, uniformly over compact intervals. Specifically,

Lemma 2.2. Assuming $K_{L}(0)=1$, we have that $K_{L}(s) \geq 1$ for all $L>0$ and $s \geq 0$. Further, for any $T$ finite there exists $B(T)<\infty$, such that $K_{L}(s) \leq 1+B(T) L^{-1}$ for all $s \leq T$ and $L \geq B(T)$. 
Proof: We first deal with the lower bound on $K_{L}(\cdot)$. To this end, fix $L>0$ and let $g(x):=1-2 x f_{L}^{\prime}(x)$. It is easy to check that $g(x)=1+4 L x(1-x)-x^{2 k} \geq 0$ for $x \in[0,1]$ and $g(x) \leq 0$ for $x \geq 1$. By Lemma 2.1, we know that the functions $R_{L}(\cdot, \cdot)$, and $C_{L}(\cdot, \cdot)$ are non negative, as is $\psi(r)$ for $r \geq 0$, so from (1.9) we get the lower bound $\partial_{s} K_{L}(s) \geq g\left(K_{L}(s)\right)$. Thus, with $\phi(x)$ a differentiable function that is strictly increasing on $[0,1]$ and such that $\phi(x)=1$ for all $x \geq 1$, we find that

$$
\partial_{s} \phi\left(K_{L}(s)\right) \geq \phi^{\prime}\left(K_{L}(s)\right) g\left(K_{L}(s)\right) \geq 0 .
$$

Consequently, $\phi\left(K_{L}(s)\right) \geq \phi\left(K_{L}(0)\right)=\phi(1)=1$ for all $s \geq 0$, implying by the choice of $\phi(\cdot)$ that $K_{L}(s) \geq 1$ for all $s \geq 0$.

Turning now to the complementary upper bound, recall that $\psi(r)$ is a polynomial of degree $m-1$, hence there exists $\kappa<\infty$ such that $\psi(a b) \leq \kappa\left(1+a^{2}\right)^{m / 2}\left(1+b^{2}\right)^{m / 2}$ for all $a, b$. Thus, by (2.1), the monotonicity of $\psi(r)$ on $\mathbb{R}_{+}$and the non-negative definiteness of $C_{L}(s, u)$ we have that for any $s, t, u \geq 0$,

$$
\psi\left(C_{L}(s, u)\right) \leq \kappa\left(1+K_{L}(u)\right)^{\frac{m}{2}}\left(1+K_{L}(s)\right)^{\frac{m}{2}}, \quad \int_{0}^{t} R_{L}(s, u) d u \leq \sqrt{t K_{L}(s)}
$$

and from (1.9) we find that

$$
\partial_{s} K_{L}(s) \leq g\left(K_{L}(s)\right)+2 \beta^{2} \kappa\left(1+\sup _{u \leq s} K_{L}(u)\right)^{m} \sqrt{K_{L}(s)} \sqrt{s} .
$$

Setting now $B(T)=1+4 \beta^{2} \kappa 3^{m} \sqrt{T}$ and fixing $T<\infty$ and $L \geq B(T)$, let

$$
\tau:=\inf \left\{u \geq 0: K_{L}(u) \geq 1+B L^{-1}\right\} .
$$

By the continuity of $K_{L}(\cdot)$ and the fact that $K_{L}(0)=1<1+B L^{-1}$, we have that $\tau>0$ and further, if $\tau<\infty$ then necessarily

$$
K_{L}(\tau)=\sup _{u \leq \tau} K_{L}(u)=1+B L^{-1} \leq 2 .
$$

Recall that $g(x) \leq 1+4 L(1-x)$ when $x \geq 1$, whereas from (2.3) we see that if $\tau<\infty$ then

$$
\partial_{s} K_{L}(s)_{\left.\right|_{s=\tau}} \leq 1-4 B+4 \beta^{2} \kappa 3^{m} \sqrt{\tau} .
$$

Recall the definition of $\tau<\infty$ implying that $\partial_{s} K_{L}(s) \geq 0$ at $s=\tau$. Hence, our choice of $B=B(T)$ results with $\tau>T$. That is, $K_{L}(s) \leq 1+B L^{-1}$ for all $s \leq T$ and $L \geq B(T)$, as claimed.

Let $\mu_{L}(s)=f_{L}^{\prime}\left(K_{L}(s)\right)$ and $h_{L}(s)=\partial_{s} K_{L}(s)$. Fixing hereafter $T<\infty$, we next prove the equicontinuity and uniform boundedness of $\left(C_{L}, R_{L}, K_{L}, \mu_{L}, h_{L}\right)$, en-route to having limit points for $\left(C_{L}, R_{L}, K_{L}\right)$.

Lemma 2.3. The continuous functions $\left(C_{L}(s, t), R_{L}(s, t)\right)$ and their derivatives are bounded uniformly in $L \geq B(T)$ and $0 \leq t \leq s \leq T$. Further, the continuous functions $\left(\mu_{L}(s), h_{L}(s)\right)$ and their derivatives are bounded uniformly in $L \geq B(T)$ and $s \in[0, T]$.

Proof: Recall that by Lemma 2.2, for any $L \geq B(T)$,

$$
\sup _{s \leq T}\left|K_{L}(s)-1\right| \leq \frac{B(T)}{L} .
$$

Consequently, the collection $\left\{C_{L}(s, t), 0 \leq t \leq s \leq T, L \geq B\right\}$ is uniformly bounded. By (2.4) and our choice of $f_{L}(r)$, we have that

$$
\left|\mu_{L}(s)\right| \leq 2 L\left|K_{L}(s)-1\right|+K_{L}(s)^{2 k-1} \leq 2 B(T)+2^{2 k-1}, \quad \forall L \geq B(T), s \leq T,
$$

whereas by (2.2) the collection $\left\{R_{L}(s, t), 0 \leq t \leq s \leq T, L \geq B\right\}$ is also uniformly bounded. Further, since

$$
h_{L}(s)=1-2 K_{L}(s) \mu_{L}(s)+2 \beta^{2} \int_{0}^{s} \psi\left(C_{L}(s, u)\right) R_{L}(s, u) d u,
$$


it follows from the uniform boundedness of $K_{L}, \mu_{L}, C_{L}$ and $R_{L}$ that $\left\{h_{L}(s), s \in[0, T], L \geq B\right\}$ is also uniformly bounded. By the same reasoning, from (1.7) and (1.8) we deduce that $\partial_{s} C_{L}(s, t)$ and $\partial_{s} R_{L}(s, t)$ are bounded uniformly in $L \geq B$ and $s, t \in[0, T]$.

Next, differentiating the identity $(2.2)$ with respect to $t$, we get for $f=f_{L}$ that

$$
\partial_{t} H_{L}(s, t)=\sum_{n \geq 1} \beta^{2 n} \sum_{\sigma \in \mathrm{NC}_{n}} \int_{t=t_{1} \leq t_{2} \cdots \leq t_{2 n} \leq s} \prod_{i \in \operatorname{cr}(\sigma)} \nu^{\prime \prime}\left(C_{L}\left(t_{i}, t_{\sigma(i)}\right)\right) \prod_{j=2}^{2 n} d t_{j},
$$

where $N C_{n}$ denotes the finite set of non-crossing involutions of $\{1, \ldots, 2 n\}$ without fixed points. With the Catalan number $\left|N C_{n}\right|$ bounded by $4^{n}$, and since $C_{L}\left(t_{i}, t_{\sigma(i)}\right) \in[0,2]$ for $t_{i}, t_{\sigma(i)} \leq T, L \geq B(T)$, we thus deduce by the monotonicity of $r \mapsto \nu^{\prime \prime}(r)$ that

$$
0 \leq \partial_{t} H_{L}(s, t) \leq \sum_{n \geq 1} \frac{\beta^{2 n}}{(2 n-1) !} 4^{n} \nu^{\prime \prime}(2)^{n}(s-t)^{2 n-1},
$$

so $\partial_{t} H_{L}(s, t)$ is finite and bounded uniformly in $L \geq B(T)$ and $0 \leq t \leq s \leq T$. Since

$$
\partial_{t} R_{L}(s, t)=\mu_{L}(t) R_{L}(s, t)-e^{-\int_{t}^{s} \mu_{L}(u) d u} \partial_{t} H_{L}(s, t),
$$

we thus have that $\left|\partial_{t} R_{L}(s, t)\right|$ is also bounded uniformly in $L \geq B(T)$ and $0 \leq t \leq s \leq T$.

In the course of proving [5, Lemma 4.1], one finds that $\partial_{t} C(s, t)=R(s, t)+D(s, t)$ for the function $D(s, t)$ of $[5,(4.2)]$. Consequently, in case of $f=f_{L}$ we have that

$\partial_{t} C_{L}(s, t)=R_{L}(s, t)-\mu_{L}(t) C_{L}(s, t)+\beta^{2} \int_{0}^{t} C_{L}(s, u) R_{L}(t, u) \nu^{\prime \prime}\left(C_{L}(t, u)\right) d u+\beta^{2} \int_{0}^{s} \nu^{\prime}\left(C_{L}(t, u)\right) R_{L}(s, u) d u$,

which of course is also bounded uniformly in $L \geq B(T)$ and $0 \leq t \leq s \leq T$.

Turning to deal with $h_{L}(\cdot)$, setting $g_{L}(r):=\left[f_{L}^{\prime}(r) r\right]^{\prime}-2 L=4 L(r-1)+k r^{2 k-1}$, we deduce from (2.4) that $\left|g_{L}\left(K_{L}(s)\right)\right| \leq 4 B(T)+k 2^{2 k}$ for any $s \leq T$ and $L \geq B(T)$. Differentiating (2.5) we find that $\partial_{s} h_{L}(s)=$ $-4 L h_{L}(s)+\kappa_{L}(s)$ for

$$
\kappa_{L}(s)=-2 g_{L}\left(K_{L}(s)\right) h_{L}(s)+2 \beta^{2} \partial_{s} \int_{0}^{s} \psi\left(C_{L}(s, u)\right) R_{L}(s, u) d u,
$$

which is thus bounded uniformly in $L \geq B(T)$ and $s \leq T$ (in view of the uniform boundedness of $h_{L}, C_{L}$, $R_{L}, \partial_{s} C_{L}$ and $\left.\partial_{s} R_{L}\right)$. Further, recall that $K_{L}(0)=1$, so by (1.9) and our choice of $f_{L}(r)$ we have that $h_{L}(0)=1-2 f_{L}^{\prime}(1)=0$, resulting with

$$
h_{L}(s)=\int_{0}^{s} e^{-4 L(s-u)} \kappa_{L}(u) d u .
$$

Since $A(T)=\sup \left\{\left|\kappa_{L}(u)\right|: L \geq B(T), u \leq T\right\}$ is finite, we thus deduce that for $L \geq B(T)$,

$$
\sup _{s \leq T}\left|h_{L}(s)\right| \leq \frac{A(T)}{4 L},
$$

and the uniform boundedness of $\left|\partial_{s} h_{L}(s)\right|$ follows.

Finally, by definition, $\partial_{s} \mu_{L}(s)=f_{L}^{\prime \prime}\left(K_{L}(s)\right) h_{L}(s)$, yielding for our choice of $f_{L}$ that

$$
\left|\partial_{s} \mu_{L}(s)\right| \leq\left(2 L+(2 k-1) 2^{2 k-3}\right)\left|h_{L}(s)\right|, \quad \forall L \geq B(T), s \leq T,
$$

which by (2.6) provides the uniform boundedness of $\left|\partial_{s} \mu_{L}(s)\right|$.

Proof of Proposition 1.1. By Lemma 2.3 we have that $\left(C_{L}(s, t), R_{L}(s, t)\right), L \geq B(T)$ are equicontinuous and uniformly bounded on $0 \leq t \leq s \leq T$. Further, $\left(K_{L}, \mu_{L}, h_{L}\right)$ are then equicontinuous and uniformly bounded on $[0, T]$. By the Arzela-Ascoli theorem, the collection $\left(C_{L}, R_{L}, K_{L}, \mu_{L}, h_{L}\right)$ thus has a limit point $(C, R, K, \mu, h)$ with respect to uniform convergence on $[0, T]$ (or $0 \leq t \leq s \leq T$, whichever is relevant). 
By Lemma 2.2 we know that the limit $K(s)=1$ for all $s \leq T$, whereas by (2.6) we have that $h(s)=0$ for all $s \leq T$. Consequently, considering (2.5) for the subsequence $L_{n} \rightarrow \infty$ for which $\left(C_{L_{n}}, R_{L_{n}}, K_{L_{n}}, \mu_{L_{n}}, h_{L_{n}}\right)$ converges to $(C, R, K, \mu, h)$ we find that the latter must satisfy (1.16). Further, since $R_{L}(t, t)=1$ and $C_{L}(t, t)=$ $K_{L}(t)$, integrating (1.7) and (1.8) we find that $R_{L}(s, t)=1+\int_{t}^{s} A_{L}(\theta, t) d \theta$ and $C_{L}(s, t)=K_{L}(t)+\int_{t}^{s} B_{L}(\theta, t) d \theta$, for

$$
\begin{aligned}
& A_{L}(\theta, t)=-\mu_{L}(\theta) R_{L}(\theta, t)+\beta^{2} \int_{t}^{\theta} R_{L}(u, t) R_{L}(\theta, u) \nu^{\prime \prime}\left(C_{L}(\theta, u)\right) d u \\
& B_{L}(\theta, t)=-\mu_{L}(\theta) C_{L}(\theta, t)+\beta^{2} \int_{0}^{\theta} C_{L}(u, t) R_{L}(\theta, u) \nu^{\prime \prime}\left(C_{L}(\theta, u)\right) d u+\beta^{2} \int_{0}^{t} \nu^{\prime}\left(C_{L}(\theta, u)\right) R_{L}(t, u) d u
\end{aligned}
$$

Since $A_{L_{n}}(s, t)$ and $B_{L_{n}}(s, t)$ converge uniformly on $0 \leq t \leq s \leq T$ to the right-hand-sides of (1.14) and (1.15), respectively, we deduce that for each limit point $(C, R, \mu)$, the functions $C(s, t)$ and $R(s, t)$ are differentiable in $s$ on $0 \leq t \leq s \leq T$ and all limit points satisfy the equations (1.14)-(1.16). Further, $C_{L}(s, t)$ are non-negative functions, and also symmetric non-negative definite kernels with $C_{L}(t, t) \rightarrow 1$. Consequently, each of their limit points corresponds to a $[0,1]$-valued symmetric non-negative kernel on $[0, T]^{2}$. Similarly, since $R_{L}(t, t)=1$ and $R_{L}(s, t)$ satisfy $(2.1)$, the same applies for any limit point $R(s, t)$. The latter are extended to functions on $[0, T]^{2}$ by setting $R(s, t)=R_{L}(s, t)=0$ whenever $s<t$.

Finally, it is not hard to verify that the system of equations $(1.14)-(1.16)$ with $C(s, t)=C(t, s), C(t, t)=$ $R(t, t)=1$ and $R(s, t)=0$ for $s<t$, admits at most one bounded solution $(R, C)$ on $[0, T]^{2}$. Indeed, considering the difference between the integrated form of (1.14)-(1.15) for two such solutions $(C, R)$ and $(\bar{C}, \bar{R})$, since $\nu^{\prime \prime}$ is uniformly Lipschitz on $[0,1]$, the functions $\Delta R(s, t)=|R(s, t)-\bar{R}(s, t)|$ and $\Delta C(s, t)=|C(s, t)-\bar{C}(s, t)|=$ $\Delta C(t, s)$ are such that for $0 \leq t \leq s \leq T$,

$$
\begin{aligned}
\Delta R(s, t) & \leq \kappa_{1}\left[\int_{t}^{s} \Delta R(v, t) d v+\int_{t}^{s} h(v) d v\right] \\
\Delta C(s, t) & \leq \kappa_{1}\left[\int_{t}^{s} \Delta C(v, t) d v+h(t)+\int_{t}^{s} h(v) d v\right]
\end{aligned}
$$

where $h(v):=\int_{0}^{v}[\Delta R(v, u)+\Delta C(v, u)] d u$ and $\kappa_{1}<\infty$ depends on $T, \beta, \nu(\cdot)$ and the maximum of $|R|$, $|C|,|\bar{R}|$ and $|\bar{C}|$ on $[0, T]^{2}$. Integrating these inequalities over $t \in[0, s]$, since $\Delta R(v, u)=0$ for $u \geq v$ and $\Delta C(v, u)=\Delta C(u, v)$, we find that

$$
\begin{aligned}
& \int_{0}^{s} \Delta R(s, t) d t \leq \kappa_{2} \int_{0}^{s} h(v) d v \\
& \int_{0}^{s} \Delta C(s, t) d t \leq \kappa_{2} \int_{0}^{s} h(v) d v
\end{aligned}
$$

for some finite constant $\kappa_{2}$ (of the same type of dependence as $\kappa_{1}$ ). Summing the latter two inequalities we see that for all $s \in[0, T]$,

$$
0 \leq h(s) \leq 2 \kappa_{2} \int_{0}^{s} h(v) d v .
$$

Further, $h(0)=0$, so by Gronwall's lemma $h(s)=0$ for all $s \in[0, T]$. Plugging this result back into (2.7)-(2.8) and observing that $\Delta R(t, t)=\Delta C(t, t)=0$, we deduce that $\Delta R(s, t)=\Delta C(s, t)=0$ for all $0 \leq t \leq s \leq T$, yielding the stated uniqueness.

In conclusion, when $L \rightarrow \infty$ the collection $\left(C_{L}, R_{L}, K_{L}\right)$ converges towards the unique solution $(C, R, K)$ of (1.14)-(1.16), as claimed.

\section{Exponential DECAY FOR SMALL VALUES OF $\beta$}

We consider here the solution $(R, C)$ of $(1.14)-(1.16)$ and prove Proposition 1.2 about its exponential decay in $s-t$ for all $\beta>0$ sufficiently small. 


\subsection{Exponential decay of $R(s, t)$ for $\beta$ small.}

Lemma 3.1. If $\beta<1 /\left(4 \sqrt{\nu^{\prime \prime}(1)}\right)$ then $R(s, t) \leq e^{-\delta_{\beta}(s-t)}$ for $\delta_{\beta}=\frac{1}{2}-2 \beta \sqrt{\nu^{\prime \prime}(1)}>0$ and all $s \geq t$.

Proof: By [12], for $s \geq t$, the solution $(R, C)$ of (1.14)-(1.15) is such that $R(s, t)=\Lambda(s, t) H(s, t)$ for $\Lambda(s, t)=$ $\exp \left(-\int_{t}^{s} \mu(u) d u\right)$ with $\mu(u)$ of $(1.16)$ and $H(s, t)$ of $(2.2)$. Further, since $C(s, t) \in[0,1]$ for all $s, t \geq 0$, it follows that $\nu^{\prime \prime}(C(s, t)) \leq \nu^{\prime \prime}(1)<\infty$. Recall that $\left|N C_{n}\right| \leq 4^{n}$, hence we deduce that

$$
H(s, t) \leq 1+\sum_{n \geq 1} \beta^{2 n} 4^{n} \nu^{\prime \prime}(1)^{n} \frac{(s-t)^{2 n}}{2 n !} \leq \sum_{k \geq 0}\left(2 \beta \sqrt{\nu^{\prime \prime}(1)}\right)^{k} \frac{(s-t)^{k}}{k !}=e^{2 \beta \sqrt{\nu^{\prime \prime}(1)}(s-t)} .
$$

By Lemma 2.1 we know that $C$ and $R$ are non-negative functions, hence $\mu(u) \geq \frac{1}{2}$ (by (1.16)), resulting with $\Lambda(s, t) \leq e^{-(s-t) / 2}$. In conclusion,

$$
R(s, t)=\Lambda(s, t) H(s, t) \leq e^{\left(2 \beta \sqrt{\nu^{\prime \prime}(1)}-\frac{1}{2}\right)(s-t)}=e^{-\delta_{\beta}(s-t)},
$$

where $\delta_{\beta}>0$ for $\beta<1 /\left(4 \sqrt{\nu^{\prime \prime}(1)}\right)$, as claimed.

\subsection{Exponential decay of $C(s, t)$ for $\beta$ small.}

Lemma 3.2. For some $\beta_{0}>0$ and any $\beta<\beta_{0}$ there exist $M_{\beta}<\infty$ and $0<\eta_{\beta}<\delta_{\beta}$ such that

$$
C(s, t) \leq M_{\beta} e^{-\eta_{\beta}|s-t|} .
$$

Proof: From equation (1.8) we get that for $s \geq t \geq 0$,

$$
C(s, t)=\Lambda(s, t)+\beta^{2} \int_{t}^{s} \Lambda(s, v) I_{1}(v, t) d v+\beta^{2} \int_{t}^{s} \Lambda(s, v) I_{2}(v, t) d v
$$

with $\Lambda(s, v)=\exp \left(-\int_{v}^{s} \mu(u) d u\right)$

$$
\begin{aligned}
& I_{1}(v, t)=\int_{0}^{v} C(u, t) R(v, u) \nu^{\prime \prime}(C(v, u)) d u, \\
& I_{2}(v, t)=\int_{0}^{t} \nu^{\prime}(C(v, u)) R(t, u) d u .
\end{aligned}
$$

For $\beta<1 /\left(4 \sqrt{\nu^{\prime \prime}(1)}\right)$ we know from Lemma 3.1 that $R(s, t) \leq e^{-\delta_{\beta}(s-t)}$. With $C(v, u)=C(u, v) \in[0,1]$ and since $\nu^{\prime}(r) \leq \nu^{\prime \prime}(1) r$ and $\nu^{\prime \prime}(r) \leq \nu^{\prime \prime}(1)$ for $0 \leq r \leq 1$, we get that for $v \geq t$,

$$
\begin{aligned}
& I_{1}(v, t) \leq \nu^{\prime \prime}(1) \int_{0}^{v} C(u, t) e^{-\delta_{\beta}(v-u)} d u, \\
& I_{2}(v, t) \leq \nu^{\prime \prime}(1) \delta_{\beta}^{-1} \sup _{u \leq t} C(u, v) .
\end{aligned}
$$

Recall that $\mu(u) \geq 1 / 2$, so $\Lambda(s, v) \leq e^{-(s-v) / 2}$. Hence, with the symmetric function $\Delta(t, s):=\sup _{u \leq t, v \leq s} C(u, v)$ we deduce from (3.1) that for $s \geq t \geq 0$,

$$
\begin{aligned}
\Delta(t, s) \leq & e^{-\frac{1}{2}(s-t)}+\beta^{2} \nu^{\prime \prime}(1) \int_{t}^{s} e^{-\frac{1}{2}(s-v)}\left[\int_{0}^{v} C(u, t) e^{-\delta_{\beta}(v-u)} d u+\delta_{\beta}^{-1} \Delta(t, v)\right] d v \\
\leq & e^{-\frac{1}{2}(s-t)}+\beta^{2} \nu^{\prime \prime}(1) \int_{t}^{s} e^{-\frac{1}{2}(s-v)} \int_{0}^{t} e^{-\delta_{\beta}(v-u)} d u d v \\
& +\beta^{2} \nu^{\prime \prime}(1) \int_{t}^{s} \Delta(t, v)\left[\delta_{\beta}^{-1} e^{-\frac{1}{2}(s-v)}+\int_{t}^{v} e^{-\frac{1}{2}(s-v)-\delta_{\beta}(v-u)} d u\right] d v
\end{aligned}
$$

It is straightforward to see that for any $\delta \in(0,1 / 2)$ and $s \geq t$,

$$
\int_{t}^{s} e^{-\frac{1}{2}(s-v)-\delta(v-t)} d v \leq 2(1-2 \delta)^{-1} e^{-\delta(s-t)}
$$


and with $\delta_{\beta} \in(0,1 / 2)$ we thus obtain for $s \geq t$ the bound

$$
\Delta(t, s) \leq M_{\beta} e^{-\delta_{\beta}(s-t)}+A_{\beta} \int_{t}^{s} \Delta(t, v) e^{-\delta_{\beta}(s-v)} d v,
$$

with $M_{\beta}=1+2 \beta^{2} \nu^{\prime \prime}(1)\left(1-2 \delta_{\beta}\right)^{-1} \delta_{\beta}^{-1}$ and $A_{\beta}=2 \beta^{2} \nu^{\prime \prime}(1) \delta_{\beta}^{-1}$. Therefore, fixing $t \geq 0$, the function $h_{t}(s)=e^{\delta_{\beta}(s-t)} \Delta(t, s)$ satisfies

$$
h_{t}(s) \leq M_{\beta}+A_{\beta} \int_{t}^{s} h_{t}(v) d v, \quad s \geq t,
$$

and so by Gronwall's lemma $h_{t}(s) \leq M_{\beta} e^{A_{\beta}(s-t)}$. We therefore conclude that for any $s \geq t$,

$$
C(s, t) \leq M_{\beta} e^{-\left(\delta_{\beta}-A_{\beta}\right)(s-t)},
$$

which proves the lemma since for $\beta \rightarrow 0$ we have that $\delta_{\beta} \rightarrow 1 / 2$ while $A_{\beta} \rightarrow 0$ (and so $\eta_{\beta}=\delta_{\beta}-A_{\beta}>0$ for any $\beta>0$ small enough).

\section{Getting the FDT equations}

With $\boldsymbol{\Gamma}=\{(s, t): 0 \leq t \leq s\} \subset \mathbb{R}_{+} \times \mathbb{R}_{+}$, we consider the map $\Psi:(R, C) \rightarrow(\widetilde{R}, \widetilde{C})$ on

$$
\mathcal{A}^{+}=\left\{(R, C) \in \mathcal{C}(\boldsymbol{\Gamma}) \times \mathcal{C}\left(\mathbb{R}_{+} \times \mathbb{R}_{+}\right): R(t, t)=C(t, t)=1, R(s, t) \geq 0, C(s, t)=C(t, s) \geq 0\right\},
$$

such that for $s \geq t$,

$$
\begin{aligned}
& \partial_{s} \widetilde{R}(s, t)=-\mu_{R, C}(s) \widetilde{R}(s, t)+\beta^{2} \int_{t}^{s} \widetilde{R}(u, t) \widetilde{R}(s, u) \nu^{\prime \prime}(C(s, u)) d u \\
& \partial_{s} \widetilde{C}(s, t)=-\mu_{R, C}(s) \widetilde{C}(s, t)+\beta^{2} \int_{0}^{s} C(u, t) R(s, u) \nu^{\prime \prime}(C(s, u)) d u+\beta^{2} \int_{0}^{t} \nu^{\prime}(C(s, u)) R(t, u) d u
\end{aligned}
$$

where $\widetilde{R}(t, t)=\widetilde{C}(t, t)=1, \widetilde{C}(t, s)=\widetilde{C}(s, t)$ and

$$
\mu_{R, C}(s)=\frac{1}{2}+\beta^{2} \int_{0}^{s} \psi(C(s, u)) R(s, u) d u .
$$

Assuming $(R, C) \in \mathcal{A}^{+}$, we have that $\mu_{R, C}(s) \geq 1 / 2$ is continuous and further, by [12] there exists a unique non-negative solution $\widetilde{R}(s, t)$ of (4.1) which is continuous on $\boldsymbol{\Gamma}$ (see for example (2.2) for existence, uniqueness and non-negativity of the solution, and the proof of Lemma 2.3 for the differentiability, hence continuity of $\widetilde{R}(s, t))$. With $C \geq 0$ and $R \geq 0$, clearly there is also a unique non-negative solution $\widetilde{C}(s, t)$ to $(4.2)$ which is continuous on $\boldsymbol{\Gamma}$ and due to the boundary condition $C(t, t)=1$, its symmetric extension to $\mathbb{R}_{+} \times \mathbb{R}_{+}$remains continuous. Thus, $\Psi\left(\mathcal{A}^{+}\right) \subseteq \mathcal{A}^{+}$.

We proceed to show that for small $\beta$ and a suitable choice of the positive constants $\delta, r, \rho, c$ the solution $(R, C)$ of (1.14)- (1.15) is a fixed point of the mapping $\Psi$ on the space

$\mathcal{S}(\delta, r, \rho, c)=\left\{(R, C) \in \mathcal{A}(\delta, r, \rho, c): \exists R_{\mathrm{fdt}}(\tau)=\lim _{t \rightarrow \infty} R(t+\tau, t), \exists C_{\mathrm{fdt}}(-\tau)=C_{\mathrm{fdt}}(\tau)=\lim _{t \rightarrow \infty} C(t+\tau, t), \forall \tau \geq 0\right\}$, where

$$
\mathcal{A}(\delta, r, \rho, c)=\left\{(R, C) \in \mathcal{A}^{+}: C(s, t) \leq c e^{-\delta|s-t|}, \quad R(s, t) \leq \rho(r|s-t|+1)^{-3 / 2} e^{-\delta(s-t)} \quad \text { for all } s \geq t\right\} .
$$

This of course implies that the solution $(R, C)$ of (1.14)- (1.15) is such that the functions

$$
\begin{aligned}
& R_{\mathrm{fdt}}(\tau)=\lim _{t \rightarrow \infty} R(t+\tau, t), \\
& C_{\mathrm{fdt}}(\tau)=\lim _{t \rightarrow \infty} C(t+\tau, t),
\end{aligned}
$$


are well defined for all $\tau \geq 0$. Further, for any $(R, C) \in \mathcal{S}(\delta, r, \rho, c)$ the corresponding functions $\left(R_{\mathrm{fdt}}, C_{\mathrm{fdt}}\right)$ are clearly in the set

$$
\begin{array}{ll}
\mathcal{B}(\delta, r, \rho, c):=\left\{(R, C) \in \mathcal{B}\left(\mathbb{R}_{+}\right) \times \mathcal{B}(\mathbb{R}): C(0)=R(0)=1,\right. & 0 \leq C(\tau)=C(-\tau) \leq c e^{-\delta|\tau|}, \\
& \left.0 \leq R(\tau) \leq \rho(r \tau+1)^{-3 / 2} e^{-\delta \tau}\right\},
\end{array}
$$

so in particular, (4.5) holds for all $\tau \in \mathbb{R}$ and $\left(R_{\mathrm{fdt}}, C_{\mathrm{fdt}}\right)$ of (4.4)-(4.5) are of exponential decay.

To this end, we start by finding constants $\delta, \rho, c$ and $r=r(\beta)$ for which $\mathcal{S}(\delta, r, \rho, c)$ is closed under the mapping $\Psi$.

Proposition 4.1. There exist finite, positive universal constants $c_{1}$ and $M_{1} \geq 2$, such that for $c=2, \rho=c_{1}$, $\delta=1 / 6$ and $r=\beta \sqrt{\nu^{\prime \prime}(c)} \leq 1 /\left(3 M_{1}\right)$, both

$$
\Psi(\mathcal{A}(\delta, r, \rho, c)) \subseteq \mathcal{A}(\delta, r, \rho, c),
$$

and

$$
\Psi(\mathcal{S}(\delta, r, \rho, c)) \subseteq \mathcal{S}(\delta, r, \rho, c) .
$$

Proof of Proposition 4.1: We first verify that (4.6) holds. To this end, setting $\widetilde{R}(s, t)=\Lambda(s, t) \widetilde{H}(s, t)$ for

$$
\Lambda(s, t)=e^{-\int_{t}^{s} \mu_{R, C}(u) d u},
$$

we have that $\widetilde{H}(t, t)=1$. Further, from [12] we have that for any $(s, t) \in \boldsymbol{\Gamma}$,

$$
\widetilde{H}(s, t)=1+\sum_{n \geq 1} \beta^{2 n} \sum_{\sigma \in \mathrm{NC}_{n}} \int_{t \leq t_{1} \cdots \leq t_{2 n} \leq s} \prod_{i \in \operatorname{cr}(\sigma)} \nu^{\prime \prime}\left(C\left(t_{i}, t_{\sigma_{i}}\right)\right) \prod_{j=1}^{2 n} d t_{j} .
$$

Consequently, as $\left|\mathrm{NC}_{n}\right|=(2 \pi)^{-1} \int_{-2}^{2} x^{2 n} \sqrt{4-x^{2}} d x$ and $C(u, v) \in[0, c]$ for $(R, C) \in \mathcal{A}(\delta, r, \rho, c)$, we have the bound

$$
\begin{aligned}
\widetilde{H}(s, t) & \leq \sum_{n \geq 0}\left(\beta^{2} \nu^{\prime \prime}(c)\right)^{n} \sum_{\sigma \in \mathrm{NC}_{n}} \int_{t \leq t_{1} \leq \cdots \leq t_{2 n} \leq s} \prod_{j=1}^{2 n} d t_{j} \\
& =\sum_{n \geq 0} \frac{\left(\beta^{2} \nu^{\prime \prime}(c)\right)^{n}(s-t)^{2 n}}{(2 n !)}(2 \pi)^{-1} \int_{-2}^{2} x^{2 n} \sqrt{4-x^{2}} d x \\
& =(2 \pi)^{-1} \int_{-2}^{2} e^{\beta \sqrt{\nu^{\prime \prime}(c)}(s-t) x} \sqrt{4-x^{2}} d x .
\end{aligned}
$$

It is well known (see for example [3, (3.8)]) that for some universal constant $1 \leq c_{1}<\infty$ and all $\theta$,

$$
(2 \pi)^{-1} \int_{-2}^{2} e^{\theta x} \sqrt{4-x^{2}} d x \leq c_{1}(1+|\theta|)^{-3 / 2} e^{2|\theta|},
$$

from which we thus deduce that

$$
\widetilde{H}(s, t) \leq c_{1}\left(1+\beta \sqrt{\nu^{\prime \prime}(c)}(s-t)\right)^{-3 / 2} e^{2 \beta \sqrt{\nu^{\prime \prime}(c)}(s-t)} .
$$

Further, since $(R, C) \in \mathcal{A}^{+}$, we know that $\mu_{R, C}(u) \geq 1 / 2$ resulting with $\Lambda(s, t) \leq e^{-(s-t) / 2}$. It then follows that for our choice of $\rho=c_{1}, r=\beta \sqrt{\nu^{\prime \prime}(c)}$ and $\delta=1 / 6 \leq 1 / 2-2 r$,

$$
\begin{aligned}
\widetilde{R}(s, t) & \leq c_{1}\left(1+\beta \sqrt{\nu^{\prime \prime}(c)}(s-t)\right)^{-3 / 2} e^{-\left(\frac{1}{2}-2 \beta \sqrt{\nu^{\prime \prime}(c)}\right)(s-t)} \\
& \leq \rho(1+r(s-t))^{-3 / 2} e^{-\delta(s-t)} .
\end{aligned}
$$


Considering next the function $\widetilde{C}$, recall that $\widetilde{C}(t, t)=1$ and for $(R, C) \in \mathcal{A}(\delta, r, \rho, c)$ we have that $\nu^{\prime}(C(v, u)) \leq \nu^{\prime \prime}(c) C(v, u)$ and $\Lambda(s, v) \leq e^{-(s-v) / 2}$ for all $v \leq s$ and $u$. Thus, we get from (4.2) that for $(s, t) \in \boldsymbol{\Gamma}$ and $(R, C) \in \mathcal{A}(\delta, r, \rho, c)$,

$$
\begin{aligned}
& \widetilde{C}(s, t)=\Lambda(s, t)+\beta^{2} \int_{t}^{s} \Lambda(s, v) d v\left[\int_{0}^{v} C(u, t) R(v, u) \nu^{\prime \prime}(C(v, u)) d u+\int_{0}^{t} \nu^{\prime}(C(v, u)) R(t, u) d u\right] \\
\leq & \Lambda(s, t)+\beta^{2} c \rho \nu^{\prime \prime}(c) \int_{t}^{s} e^{-\frac{1}{2}(s-v)} e^{-\delta(v-t)} d v\left[\int_{0}^{v}(r(v-u)+1)^{-\frac{3}{2}} d u+\int_{0}^{t}(r(t-u)+1)^{-\frac{3}{2}} d u\right] \\
\leq & {\left[1+2 K r^{-1} \beta^{2} c \rho \nu^{\prime \prime}(c) \Lambda(s, t)\left(\frac{1}{2}-\delta\right)^{-1}\right] e^{-\delta(s-t)} }
\end{aligned}
$$

where in the last inequality we have used (3.4) for $\delta=1 / 6<1 / 2$ and

$$
K:=\int_{0}^{\infty}(\theta+1)^{-\frac{3}{2}} d \theta=2 .
$$

This shows that $(\widetilde{R}, \widetilde{C}) \in \mathcal{A}(\delta, r, \rho, c)$ since our choices of $c=2, \delta=1 / 6, \rho=c_{1}$ and $r=\beta \sqrt{\nu^{\prime \prime}(c)}$, result with

$$
1+\frac{2 K c \rho \beta^{2} \nu^{\prime \prime}(c)}{\left(\frac{1}{2}-\delta\right) r}=1+12 K c_{1} r \leq 2=c,
$$

once we take $M_{1}=4 K c_{1}$ and $\beta$ sufficiently small for $r=r(\beta) \leq 1 /\left(3 M_{1}\right)$.

Our next task is to verify that (4.7) holds. That is, assuming that $(R, C) \in \mathcal{S}(\delta, r, \rho, c)$ we are to show that the limits $\left(\widetilde{R}_{\mathrm{fdt}}, \widetilde{C}_{\mathrm{fdt}}\right)$ exist for the solution $(\widetilde{R}, \widetilde{C})$ of $(4.1)-(4.3)$. To this end, recall that by (4.8), (4.9), and (3.1), for any $t \geq 0$ and $\tau \geq v \geq 0$,

$$
\begin{aligned}
\Lambda(t+\tau, t+v) & =e^{-(\tau-v) / 2} e^{-\beta^{2} \int_{v}^{\tau} I_{0}(t+u, t) d u} \\
\widetilde{R}(t+\tau, t) & =\Lambda(t+\tau, t) \widetilde{H}(t+\tau, t) \\
\widetilde{H}(t+\tau, t) & =1+\sum_{n \geq 1} \beta^{2 n} \sum_{\sigma \in \mathrm{NC}_{n}} \int_{0 \leq \theta_{1} \leq \cdots \leq \theta_{2 n} \leq \tau} \prod_{i \in \operatorname{cr}(\sigma)} \nu^{\prime \prime}\left(C\left(t+\theta_{i}, t+\theta_{\sigma(i)}\right)\right) \prod_{j=1}^{2 n} d \theta_{j} \\
\widetilde{C}(t+\tau, t) & =\Lambda(t+\tau, t)+\beta^{2} \int_{0}^{\tau} \Lambda(t+\tau, t+v) I_{1}(t+v, t) d v+\beta^{2} \int_{0}^{\tau} \Lambda(t+\tau, t+v) I_{2}(t+v, t) d v
\end{aligned}
$$

where by (4.3), (3.2) and (3.3),

$$
\begin{aligned}
& I_{0}(t+v, t)=\int_{-t}^{v} \psi(C(t+v, t+u)) R(t+v, t+u) d u \\
& I_{1}(t+v, t)=\int_{-t}^{v} C(t+u, t) R(t+v, t+u) \nu^{\prime \prime}(C(t+v, t+u)) d u \\
& I_{2}(t+v, t)=\int_{-t}^{0} \nu^{\prime}(C(t+v, t+u)) R(t, t+u) d u .
\end{aligned}
$$

Since $\psi(\cdot), \nu^{\prime \prime}(\cdot)$ and $\nu^{\prime}(\cdot)$ are continuous and $(R, C) \in \mathcal{S}(\delta, r, \rho, c)$, as $t \rightarrow \infty$ the bounded integrands in the preceding formulas converge pointwise (per fixed $u=v-\theta)$ to the corresponding expression for $\left(R_{\mathrm{fdt}}, C_{\mathrm{fdt}}\right)$. Further, by the exponential tails of $(R, C) \in \mathcal{A}(\delta, r, \rho, c)$ the integrals over $[-t,-m]$ in the formulas for $I_{0}, I_{1}$ and $I_{2}$, are uniformly in $t$ bounded by $\rho \delta^{-1} \psi(c) e^{-\delta m}$. Thus, applying bounded convergence theorem for the 
integrals over $[-m, v]$, then taking $m \rightarrow \infty$, we deduce that for each fixed $v \geq 0$,

$$
\begin{aligned}
\widehat{I}_{0} & :=\lim _{t \rightarrow \infty} I_{0}(t+v, t)=\int_{0}^{\infty} \psi\left(C_{\mathrm{fdt}}(\theta)\right) R_{\mathrm{fdt}}(\theta) d \theta, \\
\widehat{I}_{1}(v) & :=\lim _{t \rightarrow \infty} I_{1}(t+v, t)=\int_{0}^{\infty} C_{\mathrm{fdt}}(v-\theta) R_{\mathrm{fdt}}(\theta) \nu^{\prime \prime}\left(C_{\mathrm{fdt}}(\theta)\right) d \theta, \\
\widehat{I}_{2}(v) & :=\lim _{t \rightarrow \infty} I_{2}(t+v, t)=\int_{v}^{\infty} \nu^{\prime}\left(C_{\mathrm{fdt}}(\theta)\right) R_{\mathrm{fdt}}(\theta-v) d \theta .
\end{aligned}
$$

By the preceding discussion we also know that $0 \leq I_{i}(t+v, t) \leq \rho \psi(c) \delta^{-1}$ for $i=0,1,2$ and all $v, t \geq 0$. Thus, with $\Lambda(t+\tau, t+v) \in[0,1]$, by bounded convergence for each $\tau \geq v \geq 0$,

$$
\begin{aligned}
\widehat{\Lambda}(\tau-v) & :=\lim _{t \rightarrow \infty} \Lambda(t+\tau, t+v)=e^{-(\tau-v)\left(1 / 2+\beta^{2} \widehat{I}_{0}\right)}, \\
\widetilde{C}_{\mathrm{fdt}}(\tau) & :=\lim _{t \rightarrow \infty} \widetilde{C}(t+\tau, t)=\widehat{\Lambda}(\tau)+\beta^{2} \int_{0}^{\tau} \widehat{\Lambda}(\tau-v) \widehat{I}_{1}(v) d v+\beta^{2} \int_{0}^{\tau} \widehat{\Lambda}(\tau-v) \widehat{I}_{2}(v) d v .
\end{aligned}
$$

We also have that for any $n \in \mathbb{N}$, all $\sigma \in \mathrm{NC}_{n}$ and each fixed $\theta_{1}, \ldots, \theta_{2 n} \geq 0$,

$$
\lim _{t \rightarrow \infty} \prod_{i \in \operatorname{cr}(\sigma)} \nu^{\prime \prime}\left(C\left(t+\theta_{i}, t+\theta_{\sigma(i)}\right)\right)=\prod_{i \in \operatorname{cr}(\sigma)} \nu^{\prime \prime}\left(C_{\mathrm{fdt}}\left(\theta_{i}-\theta_{\sigma(i)}\right)\right),
$$

By bounded convergence, we have the convergence of the corresponding integrals over $0 \leq \theta_{1} \leq \cdots \leq \theta_{2 n} \leq \tau$. Further, the non-negative series (4.9) is dominated in $t$ by a summable series (see (4.10)), so by dominated convergence,

$$
\widetilde{H}_{\mathrm{fdt}}(\tau):=\lim _{t \rightarrow \infty} \widetilde{H}(t+\tau, t)=1+\sum_{n \geq 1} \beta^{2 n} \sum_{\sigma \in \mathrm{NC}_{n}} \int_{0 \leq \theta_{1} \leq \cdots \leq \theta_{2 n} \leq \tau} \prod_{i \in \operatorname{cr}(\sigma)} \nu^{\prime \prime}\left(C_{\mathrm{fdt}}\left(\theta_{i}-\theta_{\sigma(i)}\right)\right) \prod_{j=1}^{2 n} d \theta_{j} .
$$

It thus follows that

$$
\widetilde{R}_{\mathrm{fdt}}(\tau):=\lim _{t \rightarrow \infty} \widetilde{R}(t+\tau, t)=\widehat{\Lambda}(\tau) \widetilde{H}_{\mathrm{fdt}}(\tau),
$$

exists for each $\tau \geq 0$, which establishes our claim (4.7) (we have already shown that $\widetilde{C}_{\text {fdt }}(\tau)$ exists).

We next show that $\Psi$ is a contraction on $\mathcal{S}(\delta, r, \rho, c)$ and provide the set of equations that characterizes the functions $R_{\mathrm{fdt}}$ and $C_{\mathrm{fdt}}$ of Theorem 1.3.

Proposition 4.2. For $\delta, \rho, c$ and $r=r(\beta)$ of Proposition 4.1, if $\beta$ is small enough then $\Psi$ is a contraction on $\mathcal{A}(\delta, r, \rho, c)$ equipped with the norm

$$
\|(R, C)\|=\sup _{(s, t) \in \boldsymbol{\Gamma}}|R(s, t)|+\sup _{s, t \in \mathbb{R}_{+}}|C(s, t)|,
$$

and the solution $(R, C)$ of (1.14)-(1.15) is also the unique fixed point of $\Psi$ in $\mathcal{S}(\delta, r, \rho, c)$. Consequently, the functions $\left(R_{\mathrm{fdt}}, C_{\mathrm{fdt}}\right)$ of (4.4)-(4.5) are then the unique solution in $\mathcal{B}(\delta, r, \rho, c)$ of the FDT equations

$$
\begin{aligned}
R^{\prime}(\tau) & =-\mu R(\tau)+\beta^{2} \int_{0}^{\tau} R(\tau-\theta) R(\theta) \nu^{\prime \prime}(C(\theta)) d \theta \\
C^{\prime}(\tau) & =-\mu C(\tau)+\beta^{2} \int_{0}^{\infty} C(\tau-\theta) R(\theta) \nu^{\prime \prime}(C(\theta)) d \theta+\beta^{2} \int_{\tau}^{\infty} \nu^{\prime}(C(\theta)) R(\theta-\tau) d \theta+\mathbf{I}, \\
\mu & =\frac{1}{2}+\beta^{2} \int_{0}^{\infty} \psi(C(\theta)) R(\theta) d \theta+\mathbf{I},
\end{aligned}
$$

for $\mathbf{I}=0$. 
Proof of Proposition 4.2: Keeping $\delta, \rho, c$ and $r=r(\beta)$ as in Proposition 4.1, we first check that for any $\beta$ small enough, $\Psi$ is a contraction on $\mathcal{A}(\delta, r, \rho, c)$ equipped with the uniform norm $\|((R, C) \|$ of $(4.14)$. To this end, we consider the pairs $\left(\widetilde{R}_{i}, \widetilde{C}_{i}\right)=\Psi\left(R_{i}, C_{i}\right)$ for $\left(R_{i}, C_{i}\right) \in \mathcal{A}(\delta, r, \rho, c), i=1,2$. Denoting hereafter in short $\Delta f(s, t)=\left|f_{1}(s, t)-f_{2}(s, t)\right|$ and $\Delta f(s)=\sup _{u \leq v \leq s} \Delta f(v, u)$ when $f$ is one of the functions of interest to us, such as $R, C, \Lambda, \widetilde{H}, \widetilde{R}$ or $\widetilde{C}$, we shall show that there exist finite constants $M_{R}=M_{R}(\delta, \rho, c)$ and $M_{C}=M_{C}(\delta, \rho, c)$ such that for any finite $s$,

$$
\begin{aligned}
& \Delta \widetilde{R}(s) \leq M_{R} \beta^{2}[\Delta R(s)+\Delta C(s)], \\
& \Delta \widetilde{C}(s) \leq M_{C} \beta^{2}[\Delta R(s)+\Delta C(s)] .
\end{aligned}
$$

So, if $\beta$ is small enough for $M_{R} \beta^{2} \leq 1 / 3$ and $M_{C} \beta^{2} \leq 1 / 3$, then from (4.18) and (4.19) we deduce that

$$
\left\|\left(\widetilde{R}_{1}, \widetilde{C}_{1}\right)-\left(\widetilde{R}_{2}, \widetilde{C}_{2}\right)\right\|=\sup _{s \geq 0} \Delta \widetilde{R}(s)+\sup _{s \geq 0} \Delta \widetilde{C}(s) \leq \frac{2}{3}\left[\sup _{s \geq 0} \Delta R(s)+\sup _{s \geq 0} \Delta C(s)\right]=\frac{2}{3}\left\|\left(R_{1}, C_{1}\right)-\left(R_{2}, C_{2}\right)\right\| .
$$

In conclusion, the mapping $\Psi$ is then a contraction on $\mathcal{A}(\delta, r, \rho, c)$, since

$$
\left\|\Psi\left(R_{1}, C_{1}\right)-\Psi\left(R_{2}, C_{2}\right)\right\| \leq \frac{2}{3}\left\|\left(R_{1}, C_{1}\right)-\left(R_{2}, C_{2}\right)\right\|,
$$

for any $\left(R_{i}, C_{i}\right) \in \mathcal{A}(\delta, r, \rho, c), i=1,2$.

The challenge in deriving (4.18)-(4.19) is to get bounds that are uniform over $(s, t) \in \boldsymbol{\Gamma}$. In doing so, we use the tail estimates for $\left(R_{i}(s, t), C_{i}(s, t)\right), i=1,2$ (which hold for all functions in $\mathcal{A}(\delta, r, \rho, c)$ ), in order to improve upon the arguments of [5, proof of Proposition 4.2], where the Lipschitz bounds are derived for finite time intervals.

- The Lipschitz bound (4.18) on $\widetilde{R}$.

We rely on the formulas $(4.9)$ and $\widetilde{R}(s, t)=\widetilde{H}(s, t) \Lambda(s, t)$. Indeed, since $C_{1}$ and $C_{2}$ are [0, $\left.c\right]$-valued symmetric functions, $t_{i} \in[0, s]$ and both $\nu^{\prime \prime}(\cdot)$ and $\nu^{\prime \prime \prime}(\cdot)$ are non-negative and monotone non-decreasing, it follows that for any $n, t_{2 n} \leq s$ and $\sigma \in \mathrm{NC}_{n}$,

$$
\left|\prod_{i \in \operatorname{cr}(\sigma)} \nu^{\prime \prime}\left(C_{1}\left(t_{i}, t_{\sigma_{i}}\right)\right)-\prod_{i \in \operatorname{cr}(\sigma)} \nu^{\prime \prime}\left(C_{2}\left(t_{i}, t_{\sigma_{i}}\right)\right)\right| \leq n \nu^{\prime \prime}(c)^{n-1} \nu^{\prime \prime \prime}(c) \Delta C(s) .
$$

Thus, with $r=\beta \sqrt{\nu^{\prime \prime}(c)}$ we easily deduce from (4.9) that

$$
\begin{aligned}
\Delta \widetilde{H}(s, t) & \leq 4 \beta^{2} \nu^{\prime \prime \prime}(c)(s-t)^{2} \sum_{n \geq 1} n(2 n !)^{-1}[2 r(s-t)]^{2(n-1)} \Delta C(s) \\
& \leq 2 \beta^{2} \nu^{\prime \prime \prime}(c)(s-t)^{2} e^{2 r(s-t)} \Delta C(s) .
\end{aligned}
$$

Next, note that $\psi^{\prime}(\cdot)$ is a polynomial of non-negative coefficients, hence non-decreasing on $\mathbb{R}_{+}$. Consequently, for any $\left(R_{i}, C_{i}\right) \in \mathcal{A}(\delta, r, \rho, c)$ and all $(v, u) \in \mathbf{\Gamma}$,

$$
\Delta(\psi(C) R)(v, u) \leq \psi^{\prime}(c)\left[R_{1}(v, u) \Delta C(v, u)+C_{2}(v, u) \Delta R(v, u)\right] \leq \psi^{\prime}(c)[\rho \Delta C(v, u)+c \Delta R(v, u)] e^{-\delta(v-u)} .
$$

As $\left|e^{-x}-e^{-y}\right| \leq|x-y|$ for all $x, y \geq 0$, we thus get that

$$
\begin{aligned}
\Delta \Lambda(s, t) & \leq \beta^{2} e^{-(s-t) / 2} \int_{t}^{s} \int_{0}^{v} \Delta(\psi(C) R)(v, u) d u d v \\
& \leq \beta^{2} e^{-(s-t) / 2} \psi^{\prime}(c)[\rho \Delta C(s)+c \Delta R(s)] \int_{t}^{s} \int_{0}^{v} e^{-\delta(v-u)} d u d v \\
& \leq c_{2} \beta^{2}(s-t) e^{-(s-t) / 2}[\Delta C(s)+\Delta R(s)],
\end{aligned}
$$

for $c_{2}=\psi^{\prime}(c)(\rho+c) \delta^{-1}$. Since $\widetilde{R}(s, t)=\widetilde{H}(s, t) \Lambda(s, t)$ we now obtain from (4.11), (4.21) and (4.22) that

$$
\begin{aligned}
\Delta \widetilde{R}(s, t) & \leq \Lambda_{1}(s, t) \Delta \widetilde{H}(s, t)+\widetilde{H}_{2}(s, t) \Delta \Lambda(s, t) \\
& \leq 2 \beta^{2} \nu^{\prime \prime \prime}(c)(s-t)^{2} e^{-\left[\frac{1}{2}-2 r\right](s-t)} \Delta C(s)+c_{1} c_{2} \beta^{2}(s-t) e^{-\left[\frac{1}{2}-2 r\right](s-t)}[\Delta C(s)+\Delta R(s)]
\end{aligned}
$$


Further, as $1 / 2-2 r \geq 1 / 6$, the latter bound leads to (4.18) for the finite universal constant

$$
M_{R}:=\sup _{\theta \geq 0}\left(2 \nu^{\prime \prime \prime}(c) \theta^{2} e^{-\theta / 6}+c_{1} c_{2} \theta e^{-\theta / 6}\right) .
$$

- The Lipschitz bound (4.19) on $\widetilde{C}$.

Recall that $\widetilde{C}(s, t)$ for $(s, t) \in \boldsymbol{\Gamma}$ is given by (3.1), whereas for all $(R, C) \in \mathcal{A}(\delta, r, \rho, c)$ and $0 \leq t \leq v$,

$$
\begin{array}{r}
I_{1}(v, t)=\int_{0}^{v} C(u, t) R(v, u) \nu^{\prime \prime}(C(v, u)) d u \leq c \rho K r^{-1} \nu^{\prime \prime}(c) e^{-\delta(v-t)}, \\
I_{2}(v, t)=\int_{0}^{t} \nu^{\prime}(C(v, u)) R(t, u) d u \leq c \rho K r^{-1} \nu^{\prime \prime}(c) e^{-\delta(v-t)} .
\end{array}
$$

Further, with $c_{3}=\rho\left(\nu^{\prime \prime}(c)+c \nu^{\prime \prime \prime}(c)\right), c_{4}=c \nu^{\prime \prime}(c), c_{5}=\delta^{-1} \max \left(c_{3}, 2 c_{4}\right)$ and $c_{6}=\delta^{-1} \nu^{\prime \prime}(c) \max (\rho, 2 c)$, it is not hard to check that for $\left(R_{i}, C_{i}\right) \in \mathcal{A}(\delta, r, \rho, c)$ and $v \geq t \geq 0$,

$$
\begin{aligned}
& \Delta I_{1}(v, t) \leq c_{3} \Delta C(v) \int_{0}^{v} e^{-\delta(v-u)} d u+c_{4} \Delta R(v) \int_{0}^{v} e^{-\delta|u-t|} d u \leq c_{5}[\Delta R(v)+\Delta C(v)] \\
& \Delta I_{2}(v, t) \leq \rho \nu^{\prime \prime}(c) \Delta C(v) \int_{0}^{t} e^{-\delta(t-u)} d u+c \nu^{\prime \prime}(c) \Delta R(t) \int_{0}^{t} e^{-\delta|v-u|} d u \leq c_{6}[\Delta R(v)+\Delta C(v)]
\end{aligned}
$$

Thus, with $c_{7}=c \rho K / 3$ and $\beta^{2} \nu^{\prime \prime}(c) r^{-1}=r \leq 1 / 6$, we have from (4.22) that for $s \geq t \geq 0$,

$$
\begin{aligned}
\Delta \widetilde{C}(s, t) & \leq \Delta \Lambda(s, t)+c_{7} \int_{t}^{s} \Delta \Lambda(s, v) d v+\beta^{2} \int_{t}^{s} e^{-\frac{1}{2}(s-v)}\left[\Delta I_{1}(v, t)+\Delta I_{2}(v, t)\right] d v \\
& \leq \beta^{2} M_{C}[\Delta C(s)+\Delta R(s)],
\end{aligned}
$$

where

$$
M_{C}:=c_{2} \sup _{\theta \geq 0} \theta e^{-\theta / 2}+c_{7} c_{2} \int_{0}^{\infty} u e^{-u / 2} d u+2\left(c_{5}+c_{6}\right),
$$

and consequently, (4.19) holds.

Suppose $\delta, r, \rho, c$ are such that $\Psi$ is a contraction on $\mathcal{A}(\delta, r, \rho, c)$, hence also on its non-empty subset $\mathcal{S}(\delta, r, \rho, c)$. Starting at some $S_{0}=\left(R_{0}, C_{0}\right) \in \mathcal{S}(\delta, r, \rho, c)$ consider the sequence $S_{k}=\Psi\left(S_{k-1}\right), k=1,2, \ldots$, in $\mathcal{S}(\delta, r, \rho, c)$. Since $\Psi$ is a contraction, clearly $\left\{S_{k}\right\}$ is a Cauchy sequence for the uniform norm $\|\cdot\|$ of (4.14). Hence, $S_{k} \rightarrow S_{\infty}$ in the Banach space $\left(\mathcal{C}(\boldsymbol{\Gamma}) \times \mathcal{C}\left(\mathbb{R}_{+} \times \mathbb{R}_{+}\right)\right.$, $\left.\|\cdot\|\right)$. Note that $\mathcal{A}(\delta, r, \rho, c)$ is a closed subset of this Banach space, so $S_{\infty} \in \mathcal{A}(\delta, r, \rho, c)$. Further, fixing $\tau \geq 0$, with $|(x, y)|:=|x|+|y|$, since $S_{k} \in \mathcal{S}(\delta, r, \rho, c)$ we have that

$\lim _{T \rightarrow \infty} \sup _{t, t^{\prime} \geq T}\left|S_{\infty}(t+\tau, t)-S_{\infty}\left(t^{\prime}+\tau, t^{\prime}\right)\right| \leq 2\left\|S_{\infty}-S_{k}\right\|+\lim _{T \rightarrow \infty} \sup _{t, t^{\prime} \geq T}\left|S_{k}(t+\tau, t)-S_{k}\left(t^{\prime}+\tau, t^{\prime}\right)\right|=2\left\|S_{\infty}-S_{k}\right\|$.

Taking $k \rightarrow \infty$ we deduce that $\left\{S_{\infty}(t+\tau, t)\right\}$ is a Cauchy function from $\mathbb{R}_{+}$to $[0, \rho] \times[0, c]$, hence $S_{\infty}(t+\tau, t)$ converges as $t \rightarrow \infty$. With this applying for each $\tau \geq 0$, we see that $S_{\infty} \in \mathcal{S}(\delta, r, \rho, c)$ and further that $S_{\infty}$ is the unique fixed point of the contraction $\Psi$ on the metric space $(\mathcal{S}(\delta, r, \rho, c),\|\cdot\|)$. By our construction of $\Psi$, it follows that the fixed point $S_{\infty}=(R, C)$ of $\Psi$ satisfies (1.14)-(1.15), from which we conclude that the unique solution of the latter equations is in $\mathcal{S}(\delta, r, \rho, c)$. As noted before, this yields the existence of $R_{\mathrm{fdt}}(\tau)$ of (4.4) and $C_{\mathrm{fdt}}(\tau)$ of (4.5), such that $\left(R_{\mathrm{fdt}}, C_{\mathrm{fdt}}\right) \in \mathcal{B}(\delta, r, \rho, c)$. 
In the course of proving Proposition 4.1 we found that on $\mathcal{S}(\delta, r, \rho, c)$ the mapping $\Psi$ induces a mapping $\Psi_{\mathrm{fdt}}:\left(R_{\mathrm{fdt}}, C_{\mathrm{fdt}}\right) \rightarrow\left(\widetilde{R}_{\mathrm{fdt}}, \widetilde{C}_{\mathrm{fdt}}\right)$ such that

$$
\begin{aligned}
& \widetilde{R}_{\mathrm{fdt}}(\tau)=\widehat{\Lambda}(\tau) \sum_{n \geq 0} \beta^{2 n} \sum_{\sigma \in \mathrm{NC}_{n}} \int_{0 \leq \theta_{1} \leq \cdots \leq \theta_{2 n} \leq \tau} \prod_{i \in \operatorname{cr}(\sigma)} \nu^{\prime \prime}\left(C_{\mathrm{fdt}}\left(\theta_{i}-\theta_{\sigma(i)}\right)\right) \prod_{j=1}^{2 n} d \theta_{j}, \\
& \widetilde{C}_{\mathrm{fdt}}(\tau)=\widehat{\Lambda}(\tau)+\beta^{2} \int_{0}^{\tau} \widehat{\Lambda}(\tau-v) \widehat{I}_{1}(v) d v+\beta^{2} \int_{0}^{\tau} \widehat{\Lambda}(\tau-v) \widehat{I}_{2}(v) d v
\end{aligned}
$$

where $\widehat{\Lambda}(\tau)=e^{-\mu \tau}$ for $\mu=1 / 2+\beta^{2} \widehat{I}_{0}$ of (4.17), while $\widehat{I}_{1}(v)$ and $\widehat{I}_{2}(v)$ of (4.12)-(4.13) are the two integrals on the right-hand-side of (4.16). In particular, $\widetilde{R}_{\mathrm{fdt}}$ and $\widetilde{C}_{\mathrm{fdt}}$ are differentiable on $\mathbb{R}_{+}$, and by [12] we have that for $\tau \geq 0$,

$$
\widetilde{R}_{\mathrm{fdt}}^{\prime}(\tau)=-\mu \widetilde{R}_{\mathrm{fdt}}(\tau)+\beta^{2} \int_{0}^{\tau} \widetilde{R}_{\mathrm{fdt}}(\tau-\theta) \widetilde{R}_{\mathrm{fdt}}(\theta) \nu^{\prime \prime}\left(C_{\mathrm{fdt}}(\theta)\right) d \theta
$$

with $\widetilde{R}_{\mathrm{fdt}}(0)=1$, while

$$
\widetilde{C}_{\mathrm{fdt}}^{\prime}(\tau)=-\mu \widetilde{C}_{\mathrm{fdt}}(\tau)+\beta^{2} \widehat{I}_{1}(\tau)+\beta^{2} \widehat{I}_{2}(\tau),
$$

with $\widetilde{C}_{\mathrm{fdt}}(0)=1$. Since the solution $(R, C)$ of (1.14)-(1.15) is a fixed point of $\Psi$, the corresponding pair $\left(R_{\mathrm{fdt}}, C_{\mathrm{fdt}}\right)$ is a fixed point of $\Psi_{\mathrm{fdt}}$, which by (4.23)-(4.24) satisfies the FDT equations (4.15)-(4.17) with $\mathbf{I}=0$.

Recall that $\mathcal{B}(\delta, r, \rho, c)$ consists of pairs $(R, C)$ of functions that are uniformly bounded, exponentially decaying to zero at infinity, and of fixed values at zero. It is not hard to verify that when $\mathbf{I}=0$, any solution of (4.15)-(4.17) in this space, is a fixed point of $\Psi_{\mathrm{fdt}}$. Further, $\Psi_{\mathrm{fdt}}$ is a contraction on $\mathcal{B}(\delta, r, \rho, c)$, equipped with the supremum norm, since following the very same arguments we used in proving the Lipschitz estimates (4.18)-(4.19) of Proposition 4.2, we find that $\Delta \widetilde{R}_{\mathrm{fdt}}(\tau) \leq M_{R} \beta^{2}\left[\Delta R_{\mathrm{fdt}}(\tau)+\Delta C_{\mathrm{fdt}}(\tau)\right]$ and $\Delta \widetilde{C}_{\mathrm{fdt}}(\tau) \leq$ $M_{C} \beta^{2}\left[\Delta R_{\mathrm{fdt}}(\tau)+\Delta C_{\mathrm{fdt}}(\tau)\right]$ for all $\tau<\infty$, now with $\Delta f(\tau)=\sup _{\theta \leq \tau}|f(\theta)|$. This of course proves the uniqueness of the solution of (4.15)-(4.17) in $\mathcal{B}(\delta, r, \rho, c)$, in case $\mathbf{I}=0$, as claimed.

\section{Study of the FDT EQUations}

In this section, we complete the proof of Theorem 1.3 by relating the solutions of the equations (4.15)-(4.17) with the solution of (1.20).

Specifically, we first prove Proposition 1.4 about existence, uniqueness, limiting value and exponential convergence of the solution of (1.20). Fixing $\beta<\beta_{c}$, we then know that the unique solution of (1.20) for $b=1 / 2$ and $\phi(x)=b+2 \beta^{2} \nu^{\prime}(x)$, exists, is twice continuously differentiable, positive, and of negative derivative, such that both converge exponentially fast to zero when $s \rightarrow \infty$, with positive exponent $\epsilon_{\beta}=\frac{1}{2}-2 \beta^{2} \nu^{\prime \prime}(0)$. Further, for $\beta<\beta_{1} \leq \beta_{c}$ sufficiently small, by Proposition 4.2, the pair of functions $(R(\tau+t, t), C(\tau+t, t))$ converges for $t \rightarrow \infty$ to the unique solution $\left(R_{\mathrm{fdt}}(\tau), C_{\mathrm{fdt}}(\tau)\right)$ of (4.15)-(4.17), with $\mathbf{I}=0$. Hence, our next proposition (whose proof is provided at the end of the section), completes the proof of the theorem by showing that for any $\beta$, the solution $\left(C_{\mathrm{fdt}}, R_{\mathrm{fdt}}\right)$ of (4.15)-(4.17) can be expressed in terms of the solution of (1.20) for $b=1 / 2$ and $\phi(x)=\gamma+2 \beta^{2} \nu^{\prime}(x)$, provided the constant $\gamma$ is chosen accordingly. That is, with $\mathbf{I}_{\gamma}$ matching the constant $\mathbf{I}$ of (4.17) while $\phi(\cdot)$ satisfies the condition (1.21) of Proposition 1.4. Indeed, if $\beta<\beta_{c}$, then for $\gamma=b$ we have that $D_{\infty}=0, \mathbf{I}_{\gamma}=0$ and further, the resulting solution $(R, C)$ has the exponential decay property of Proposition 4.2 provided $\beta$ is small enough (for the condition $b+2 \beta^{2} \nu^{\prime}(1)>2 \sqrt{b 2 \beta^{2} \nu^{\prime \prime}(1)}$ of Proposition 1.4 to hold).

Proposition 5.1. Suppose $D(s)$ is a positive, twice continuously differentiable and decreasing solution of the equation (1.20) for $b>0, D_{\infty} \geq 0$ and $\phi(\cdot)$ which satisfy the conditions of Proposition 1.4. Then, 
$R(s)=-b^{-1} D^{\prime}(s) \geq 0, C(s)=C(-s)=D(s) \geq 0$ and $\mu=\phi(1)>0$ are such that $R(0)=C(0)=1$ and for any $\gamma \in \mathbb{R}$,

$$
\begin{aligned}
R^{\prime}(\tau) & =-\mu R(\tau)+b \int_{0}^{\tau} R(\tau-\theta) R(\theta) \phi^{\prime}(C(\theta)) d \theta, \\
C^{\prime}(\tau) & =-\mu C(\tau)+b \int_{0}^{\infty} C(\tau-\theta) R(\theta) \phi^{\prime}(C(\theta)) d \theta+b \int_{\tau}^{\infty}[\phi(C(\theta))-\gamma] R(\theta-\tau) d \theta+\mathbf{I}_{\gamma} \\
\mu & =b+b \int_{0}^{\infty} \widehat{\psi}_{\gamma}(C(\theta)) R(\theta) d \theta+\mathbf{I}_{\gamma},
\end{aligned}
$$

for $\widehat{\psi}_{\gamma}(x)=x \phi^{\prime}(x)+\phi(x)-\gamma$ and $\mathbf{I}_{\gamma}=\gamma-b+D_{\infty}\left(\phi\left(D_{\infty}\right)-\gamma\right)\left(\right.$ recall also that $\left.b=\phi\left(D_{\infty}\right)\left(1-D_{\infty}\right)\right)$.

Proof of Proposition 1.4. Let $I=\left[0,1-D_{\infty}\right]$ and $M_{\infty}\left(\mathbb{R}_{+}, I\right)$ denote the space of continuous $I$-valued functions on $\mathbb{R}_{+}$which take the value $1-D_{\infty}$ at time zero. Set $\varphi(x)=\phi\left(x+D_{\infty}\right)-\phi\left(D_{\infty}\right)$ and for any $E \in M_{\infty}\left(\mathbb{R}_{+}, I\right)$, let $k(s)=b \varphi^{\prime}(E(s))$ and

$$
H_{s}(E)=1+\sum_{n \geq 1} \sum_{\sigma \in N C_{n}} \int_{0 \leq t_{1} \cdots \leq t_{2 n} \leq s} \prod_{i \in \operatorname{cr}(\sigma)} k\left(t_{\sigma(i)}-t_{i}\right) \prod_{i=1}^{2 n} d t_{i} .
$$

Note that the continuously differentiable $s \mapsto H_{s}(E)$ is the solution of

$$
\frac{d H_{s}(E)}{d s}=b \int_{0}^{s} \varphi^{\prime}(E(s-v)) H_{s-v}(E) H_{v}(E) d v, \quad H_{0}(E)=1 .
$$

For $\mu=\phi(1)$ let

$$
\Phi(E)(s)=\left(1-D_{\infty}-b \int_{0}^{s} e^{-\mu v} H_{v}(E) d v\right) \vee 0 .
$$

Since $b \varphi^{\prime}$ is non-negative on the interval $I$, it follows that $H_{s}(E)$ is non-negative and consequently, $s \mapsto \Phi(E)(s)$ is non-increasing and also belongs to $M_{\infty}\left(\mathbb{R}_{+}, I\right)$. Further, since $\varphi^{\prime}$ is by assumption Lipschitz continuous on the compact set $I$ it follows from (5.4) that for each $T<\infty$ there exists $\kappa_{1}(T)<\infty$ such that for all $E, \bar{E} \in M_{\infty}\left(\mathbb{R}_{+}, I\right)$ and $u \leq T$,

$$
\left|H_{u}(E)-H_{u}(\bar{E})\right| \leq \kappa_{1}(T) \int_{0}^{u}|E(v)-\bar{E}(v)| d v
$$

With $y \mapsto y \vee 0$ Lipschitz continuous, this implies that for some finite, non-decreasing $\kappa_{2}(T) \geq 1$, all $E, \bar{E} \in$ $M_{\infty}\left(\mathbb{R}_{+}, I\right)$ and $s \leq T$,

$$
|\Phi(E)(s)-\Phi(\bar{E})(s)| \leq \kappa_{2}(T) \int_{0}^{s}|E(v)-\bar{E}(v)| d v .
$$

Thus, $\Phi(\cdot)$ is a contraction on $M_{\infty}\left(\mathbb{R}_{+}, I\right)$ equipped with the weighted $L_{1}$-norm $\|E\|_{*}:=\int_{0}^{\infty}|E(s)| w(s) d s$ for weight function $w(s)=\exp \left(-2 \int_{0}^{s} \kappa_{2}(u) d u\right)>0$. Consequently, we deduce that $\Phi(E)=E$ has a unique solution in $M_{\infty}\left(\mathbb{R}_{+}, I\right)$, denoted hereafter by $E_{*}(s)$. Let $\sigma_{*}>0$ be the first $s>0$ where $\Phi\left(E_{*}\right)(s)=0$. Note that the function $s \mapsto \Phi\left(E_{*}\right)(s)$ is continuously differentiable on the interval $\left[0, \sigma_{*}\right)$, in which case also

$$
E_{*}(s)=1-D_{\infty}-b \int_{0}^{s} e^{-\mu v} H_{v}\left(E_{*}\right) d v,
$$

is twice differentiable, with $E_{*}^{\prime}(s)=-b e^{-\mu s} H_{s}\left(E_{*}\right)$. Hence, applying (5.5) for $H_{s}\left(E_{*}\right)$, we have that for $s \in\left[0, \sigma_{*}\right)$,

$$
E_{*}^{\prime \prime}(s)=-\mu E_{*}^{\prime}(s)-\int_{0}^{s} \varphi^{\prime}\left(E_{*}(s-v)\right) E_{*}^{\prime}(s-v) E_{*}^{\prime}(v) d v .
$$


Integrating this equation we find that

$$
E_{*}^{\prime}(s)=E_{*}^{\prime}(0)-\int_{0}^{s}\left[\mu+\varphi\left(E_{*}(s-v)\right)-\varphi\left(E_{*}(0)\right)\right] E_{*}^{\prime}(v) d v .
$$

Since $E_{*}^{\prime}(0)=-b, E_{*}(0)=1-D_{\infty}$ and $\mu-\varphi\left(E_{*}(0)\right)=\phi\left(D_{\infty}\right)$, it follows that on $\left[0, \sigma_{*}\right)$

$$
E_{*}^{\prime}(s)=-b-\int_{0}^{s} \phi\left(E_{*}(s-v)+D_{\infty}\right) E_{*}^{\prime}(v) d v, \quad \mathbb{E}_{*}(0)=1-D_{\infty} .
$$

We next show that $\sigma_{*}=\infty$, which in view of (5.6) yields that $D(s)=E_{*}(s)+D_{\infty}$ is a twice differentiable, strictly decreasing $\left[D_{\infty}, 1\right]$-valued solution of $(1.20)$. To this end, note that $H_{s}\left(E_{*}\right) \geq 1$ since $E_{*} \in M_{\infty}\left(\mathbb{R}_{+}, I\right)$ (see (5.4)). Consequently, for all $v<\sigma_{*}$ both $E_{*}^{\prime}(v) \leq-b e^{-\mu v}<0$ and

$$
-\phi\left(E_{*}(s-v)+D_{\infty}\right) E_{*}^{\prime}(v) \geq-\phi\left(D_{\infty}\right) E_{*}^{\prime}(v) .
$$

Thus, from (5.6) we have that for $s<\sigma_{*}$,

$$
-b e^{-\mu s} \geq E_{*}^{\prime}(s) \geq-b-\phi\left(D_{\infty}\right)\left(E_{*}(s)-E_{*}(0)\right)=-\phi\left(D_{\infty}\right) E_{*}(s),
$$

since $b=\phi\left(D_{\infty}\right)\left(1-D_{\infty}\right)=\phi\left(D_{\infty}\right) E_{*}(0)$ by the definition of $D_{\infty}$. If $\sigma_{*}<\infty$, then as $s \uparrow \sigma_{*}$ we have by the continuity of $E_{*}(\cdot)$ that $E_{*}(s) \rightarrow E_{*}\left(\sigma_{*}\right)=\Phi\left(E_{*}\right)\left(\sigma_{*}\right)=0$, in contradiction with (5.7).

The uniqueness of the solution to (1.20) follows from the uniqueness of the preceding $E_{*}(\cdot)$. Indeed, if a continuously differentiable $\left[D_{\infty}, 1\right]$-valued $D(s)$ solves $(1.20)$, then $E(s)=D(s)-D_{\infty} \in M_{\infty}\left(\mathbb{R}_{+}, I\right)$ and since $b=\phi\left(D_{\infty}\right) E(0)$ we have that

$$
E^{\prime}(s)=-\phi\left(D_{\infty}\right) E(s)-\int_{0}^{s} \varphi(E(s-v)) E^{\prime}(v) d v, \quad E(0)=1-D_{\infty} .
$$

Hence, $E(s)$ is twice differentiable, with $E^{\prime}(0)=-b$, and differentiating (5.8) we get that

$$
E^{\prime \prime}(s)=-\mu E^{\prime}(s)-\int_{0}^{s} \varphi^{\prime}(E(s-v)) E^{\prime}(s-v) E^{\prime}(v) d v
$$

(using the fact that $\varphi(E(0))+\phi\left(D_{\infty}\right)=\mu$ ). Thus, $H_{s}(E)=-b^{-1} e^{-\mu s} E^{\prime}(s)$ solves (5.5). This means that for all $s \geq 0$,

$$
E(s)=1-D_{\infty}-b \int_{0}^{s} e^{-\mu v} H_{v}(E) d v .
$$

Since $E(s) \geq 0$, so is the right-hand-side of $(5.10)$, that is $E=\Phi(E)$. Since $\Phi(\cdot)$ has a unique fixed point in $M_{\infty}\left(\mathbb{R}_{+}, I\right)$, the solution of $(1.20)$ must also be unique.

Next, the monotone and bounded function $D$ converges to some $x \in\left[D_{\infty}, 1\right]$. Fix $M=M_{\epsilon}$ such that $D(v) \leq x+\epsilon$ for all $v \geq M$. Then, for $s \geq 2 M$ we have that

$$
\begin{aligned}
\int_{0}^{s} \phi(D(v)) D^{\prime}(s-v) d v & =\int_{0}^{M} \phi(D(v)) D^{\prime}(s-v) d v+\int_{M}^{s} \phi(D(v)) D^{\prime}(s-v) d v \\
& \geq-\phi(1)(D(s-M)-D(s))-\phi(x+\epsilon)(1-D(s-M)) \\
& \geq-\phi(1)(x+\epsilon-x)-\phi(x+\epsilon)(1-x) .
\end{aligned}
$$

Hence, for all $s \geq 2 M$,

$$
D^{\prime}(s) \leq-b+\phi(1) \epsilon+\phi(x+\epsilon)(1-x) .
$$

Since $D(s)$ is bounded below, it follows that $\phi(1) \epsilon+\phi(x+\epsilon)(1-x) \geq b$. Taking $\epsilon \downarrow 0$ we see that $x=D_{\infty}$ (since $x \geq D_{\infty}$ and $D_{\infty}$ is the largest $y \in[0,1]$ for which $\phi(y)(1-y) \geq b$ ). 
Finally, recall that the function $E(s)=D(s)-D_{\infty}$ is strictly positive, monotone decreasing with $E^{\prime}(s)=$ $-b e^{-\mu s} H_{s}(E)$ for $H_{s}(E)$ of $(5.4)$. Since there $k(s)=b \varphi^{\prime}(E(s)) \leq b \phi^{\prime}(1)$ (by the assumed convexity of $\phi(\cdot)$ ), the same argument as in (4.10) yields that for all $s \geq 0$,

$$
H_{s}(E) \leq(2 \pi)^{-1} \int_{-2}^{2} e^{\sqrt{b \phi^{\prime}(1)} s x} \sqrt{4-x^{2}} d x \leq c_{1} e^{2 \sqrt{b \phi^{\prime}(1)} s} .
$$

Consequently, if $\phi(1)>2 \sqrt{b \phi^{\prime}(1)}$, then both $E^{\prime}(s)$ and $E(s)$ converge exponentially to zero for $s \rightarrow \infty$.

Remark. We note in passing that $\varphi(x) \geq \varphi^{\prime}(0) x$ for all $x \in[0,1]$ when $\phi$ is convex, in which case by the monotonicity of $E(s)$,

$$
J(s):=E(s)^{-1} \int_{0}^{s}\left[-E^{\prime}(v)\right] \varphi(E(s-v)) d v \geq\left(x^{-1} \varphi\right)(E(s))[E(0)-E(s)] \geq \varphi^{\prime}(0)\left[1-D_{\infty}-E(s)\right] .
$$

Further, recall that from (5.8),

$$
\frac{d \log E}{d s}(s)=\frac{E^{\prime}}{E}(s)=J(s)-\phi\left(D_{\infty}\right),
$$

hence in case $\phi\left(D_{\infty}\right)=\phi^{\prime}\left(D_{\infty}\right)\left(1-D_{\infty}\right)$, we find that $E(s) \geq E(0) \exp \left(-\phi^{\prime}\left(D_{\infty}\right) \int_{0}^{s} E(u) d u\right)$, which thus does not converge to zero exponentially fast as $s \rightarrow \infty$.

Proof of Proposition 5.1. Since $D(0)=1$ and $D(\theta) \downarrow D_{\infty}$, for $R=-b^{-1} D^{\prime}$ and any $\gamma \in \mathbb{R}$ we have that

$$
\gamma b \int_{0}^{\infty} R(\theta) d \theta=\gamma\left(1-D_{\infty}\right)=\mathbf{I}_{\gamma}-\mathbf{I}_{0},
$$

hence we may and shall assume hereafter that $\gamma=0$. Further, it is easy to check that for $\widehat{\psi}_{0}(x)=[x \phi(x)]^{\prime}$

$$
\int_{0}^{\infty} \widehat{\psi}_{0}(D(\theta)) D^{\prime}(\theta) d \theta=D_{\infty} \phi\left(D_{\infty}\right)-D(0) \phi(D(0)),
$$

so the choices of $\mu=\phi(1)=\phi(D(0))$ and $\mathbf{I}_{0}=-b+D_{\infty} \phi\left(D_{\infty}\right)$ guarantee that $C=D$ and $R=-b^{-1} D^{\prime}$ satisfy (5.3). Recall that while proving Proposition 1.4 we have seen that $D^{\prime}(0)=-b$ and for all $\tau \geq 0$

$$
D^{\prime \prime}(\tau)=-\mu D^{\prime}(\tau)-\int_{0}^{\tau} D^{\prime}(\tau-\theta) D^{\prime}(\theta) \phi^{\prime}(D(\theta)) d \theta
$$

(c.f. (5.9)), from which it immediately follows that $C=D$ and $R=-b^{-1} D^{\prime}$ satisfy (5.1).

Turning to the remaining task of verifying that (5.2) holds, note that since $D(\cdot)$ is bounded and converges to $D_{\infty}$,

$$
\int_{\tau}^{\infty} D(\theta-\tau) D^{\prime}(\theta) \phi^{\prime}(D(\theta)) d \theta+\int_{\tau}^{\infty} \phi(D(\theta)) D^{\prime}(\theta-\tau) d \theta=\phi\left(D_{\infty}\right) D_{\infty}-\phi(D(\tau)) D(0) .
$$

Similarly,

$$
\int_{0}^{\tau} D(\tau-\theta) D^{\prime}(\theta) \phi^{\prime}(D(\theta)) d \theta-\int_{0}^{\tau} \phi(D(\theta)) D^{\prime}(\tau-\theta) d \theta=\phi(D(\tau)) D(0)-\phi(D(0)) D(\tau) .
$$

Hence, using the symmetry $C(s)=C(-s)$, upon substituting in (5.2) $C=D, R=-b^{-1} D^{\prime}$ and the preceding two identities, it is not hard to verify that (5.2) holds for $\gamma=0$ if

$$
D^{\prime}(\tau)=-\phi\left(D_{\infty}\right) D_{\infty}+\mathbf{I}_{0}-\int_{0}^{\tau} \phi(D(\theta)) D^{\prime}(\tau-\theta) d \theta,
$$

which in view of (1.20) is merely the statement that $b=\phi\left(D_{\infty}\right) D_{\infty}-\mathbf{I}_{0}$. Our choice of $\mathbf{I}_{0}$ guarantees that the latter identity applies, thus completing the proof of the proposition. 


\section{About the FDT Regime AT ALL temperatures}

We return to the equations (1.15)-(1.16) and explain why (1.20) is the natural candidate for describing the function $C_{\mathrm{fdt}}(\cdot)$ for all $\beta$. To this end, set for $s \geq t$,

$$
G(s, t)=R(s, t)-2 \partial_{t} C(s, t)
$$

and

$$
I(s, t)=\beta^{2} \int_{0}^{t}\left[C(t, u) G(s, u) \nu^{\prime \prime}(C(s, u))+\nu^{\prime}(C(s, u)) G(t, u)\right] d u-2 \beta^{2} \nu^{\prime}(C(s, 0)) C(t, 0) .
$$

Since $\psi(x)=\left[x \nu^{\prime}(x)\right]^{\prime}$ and $C(s, s)=1$, it follows that

$$
\begin{aligned}
I(s, s) & =\beta^{2} \int_{0}^{s} \psi(C(s, u)) G(s, u) d u-2 \beta^{2} \nu^{\prime}(C(s, 0)) C(s, 0) \\
& =\beta^{2} \int_{0}^{s} \psi(C(s, u)) R(s, u) d u-2 \beta^{2} \nu^{\prime}(1)=\mu-\frac{1}{2}-2 \beta^{2} \nu^{\prime}(1)
\end{aligned}
$$

for $\mu$ of (1.16). Further, by similar reasoning,

$$
\begin{aligned}
2 \beta^{2} \nu^{\prime}(C(s, t))+I(s, t) & =2 \beta^{2} \int_{0}^{t} \partial_{u}\left[C(t, u) \nu^{\prime}(C(s, u))\right] d u+2 \beta^{2} \nu^{\prime}(C(s, 0)) C(t, 0)+I(s, t) \\
& =\beta^{2} \int_{0}^{t}\left[C(t, u) R(s, u) \nu^{\prime \prime}(C(s, u))+\nu^{\prime}(C(s, u)) R(t, u)\right] d u
\end{aligned}
$$

Thus, in these notations (1.14)-(1.16) are equivalent to having for $s \geq t$,

$$
\begin{aligned}
& \partial_{s} R(s, t)=-[\rho+I(s, s)] R(s, t)+\beta^{2} \int_{t}^{s} R(s, u) R(u, t) \nu^{\prime \prime}(C(s, u)) d u \\
& \partial_{s} C(s, t)=-[\rho+I(s, s)] C(s, t)+\beta^{2} \int_{t}^{s} C(u, t) R(s, u) \nu^{\prime \prime}(C(s, u)) d u+2 \beta^{2} \nu^{\prime}(C(s, t))+I(s, t)
\end{aligned}
$$

with $R(t, t)=C(t, t)=1$ and $\rho=2^{-1}+2 \beta^{2} \nu^{\prime}(1)$.

We note in passing that $G(s, t)$ is such that for $s \geq t$,

$$
\begin{aligned}
\partial_{s} G(s, t) & =-2 \partial_{t} I(s, t)-[\rho+I(s, s)] G(s, t)+2 \beta^{2} \nu^{\prime \prime}(C(s, t)) G(s, t) \\
& +\beta^{2} \int_{t}^{s} G(u, t)\left[G(s, u) \nu^{\prime \prime}\left(C(s, u)+2 \partial_{u} C(s, u) \nu^{\prime \prime}(C(s, u))\right] d u .\right.
\end{aligned}
$$

The physics prediction is that $G(t+\tau, t) \rightarrow 0$ as $t \rightarrow \infty$ while $\tau$ is fixed, for any finite $\beta$ (this is the famous FDT relation). As a result, comparing $I(s, t)$ and $I(t, t)$, we further expect that $I(t+\tau, t) \rightarrow \widehat{\mathbf{I}}$ when $t \rightarrow \infty$ while $\tau$ is fixed. We next show that the latter ansatz results with the existence of an FDT solution $\left(R_{\mathrm{fdt}}, C_{\mathrm{fdt}}\right)$ such that $R_{\mathrm{fdt}}=-2 C_{\mathrm{fdt}}^{\prime}$ and $C_{\mathrm{fdt}}$ solves $(1.20)$ for $b=1 / 2$ and $\phi(x)=\gamma+2 \beta^{2} \nu^{\prime}(x)$, where $\gamma=\widehat{\mathbf{I}}+1 / 2$.

Proposition 6.1. Assume that given the continuous function $I(s, t)$ there exists a continuously differentiable solution $\left(R_{I}(s, t), C_{I}(s, t)\right), s \geq t \geq 0$, of (6.2)-(6.3) with $\rho=2^{-1}+2 \beta^{2} \nu^{\prime}(1)$, the initial conditions $R(t, t)=$ $C(t, t)=1$ and uniformly bounded $C(s, t)$. Further, suppose that for any $T<\infty$

$$
\lim _{t \rightarrow \infty} \sup _{\tau \in[0, T]}|I(t+\tau, t)-\widehat{\mathbf{I}}|=0
$$

where the constant $\widehat{\mathbf{I}}$ is such that

$$
\sup _{0 \leq x \leq 1}\left\{\left(\widehat{\mathbf{I}}+\frac{1}{2}+2 \beta^{2} \nu^{\prime}(x)\right)(1-x)\right\} \geq \frac{1}{2}
$$


Then, $C_{I}(t+\tau, t) \rightarrow C_{\mathrm{fdt}}(\tau)$ as $t \rightarrow \infty$, uniformly in $\tau \in[0, T]$, where $C_{\mathrm{fdt}}(\tau)$ is the unique solution of (1.20) for $b=1 / 2$ and $\phi(x)=\gamma+2 \beta^{2} \nu^{\prime}(x)$, with $\gamma=\widehat{\mathbf{I}}+1 / 2$. Further, $R_{I}(t+\tau, t) \rightarrow R_{\mathrm{fdt}}(\tau)=-2 C_{\mathrm{fdt}}^{\prime}(\tau)$ as $t \rightarrow \infty$, uniformly in $\tau \in[0, T]$.

Remark. In Theorem 1.3 we circumvent the difficulty of showing the ansatz that $I(t+\tau, t)$ converges to a ( $\beta$-dependent) constant at any value of $\beta$, by verifying that at very high temperature, i.e. sufficiently small $\beta$, the exponential decay to zero in $s-t$ of $(R, C)$ results with the convergence to zero of $I$. Proposition 6.1 shows that this is the only obstacle to extending our results about the FDT regime to all temperatures.

Before proving the proposition, consider its consistency with our choice of $\gamma(\beta)$ for $\beta \geq \beta_{c}$ based on $q(\beta)$ of (1.24). Specifically, considering in (6.1) the contribution to the integral from $u \in[s-M, s]$ with $M<\infty$ arbitrarily large, we expect the FDT solution $\left(R_{\mathrm{fdt}}, C_{\mathrm{fdt}}\right)$ to contribute $-2 \beta^{2} q(\beta) \nu^{\prime}(q(\beta))$ to the limiting constant $\widehat{\mathbf{I}}=\gamma(\beta)-1 / 2$ (for which (6.6) holds by our choice of $\gamma(\beta)$ ). We further expect $\widehat{\mathbf{I}}$ to be the sum of this FDT contribution and a non-negative contribution from the aging regime (i.e. the integral over $u \in[0, s-M])$. Given the relation between $\mu$ and $\mathbf{I}_{\gamma}$ in Proposition 5.1, we deduce that $\mathbf{I}_{\gamma}$ is exactly the contribution of the aging regime to $\widehat{\mathbf{I}}$. In conclusion, we should have $\mathbf{I}_{\gamma}=0$ for $\beta<\beta_{c}, \mathbf{I}_{\gamma} \geq 0$ at $\beta=\beta_{c}$ and $\mathbf{I}_{\gamma}>0$ for $\beta>\beta_{c}$. This is indeed the case, for when $\beta<\beta_{c}$ we have $\gamma=b=1 / 2$ and $D_{\infty}=0$ leading to $\mathbf{I}_{\gamma}=0$ while for $\beta \geq \beta_{c}$, since $\nu^{\prime}(x) \leq x \nu^{\prime \prime}(x)$ for $x \geq 0$ with strict inequality when $x>0$, our choice of $\gamma(\beta)$ leads to

$$
2 I_{\gamma}=4 \beta^{2}(1-q(\beta))\left[\nu^{\prime \prime}(q(\beta))-\nu^{\prime}(q(\beta))\right]-1 \geq 4 \beta^{2}(1-q(\beta))^{2} \nu^{\prime \prime}(q(\beta))-1 \geq 0,
$$

with a strict inequality whenever $q(\beta)>0$ (in particular, for all $\beta>\beta_{c}$ ).

Proof of Proposition 6.1. Fixing hereafter the values of $\beta$ and $\rho=1 / 2+2 \beta^{2} \nu^{\prime}(1)$ and the initial conditions $R(t, t)=C(t, t)=1$, a re-run of the argument at the end of the proof of Proposition 1.1 shows that per given continuous function $I(s, t)$, the system of equations (6.2)-(6.3) admits at most one bounded solution, denoted $\left(R_{I}(s, t), C_{I}(s, t)\right)$ on any compact interval $0 \leq t \leq s \leq T$. Consequently, it has at most one continuous solution $\left(R_{I}, C_{I}\right)$ for all $s \geq t \geq 0$.

In particular, in case $I(s, t)=\widehat{\mathbf{I}}$ is a constant that satisfies (6.6), we know that (1.21) holds for $b=1 / 2$, $\gamma=\widehat{\mathbf{I}}+1 / 2$ and $\phi(x)=\gamma+2 \beta^{2} \nu^{\prime}(x)$. With $D(\tau)$ denoting the unique solution of (1.20) for these parameters, we claim that $R_{\widehat{\mathbf{I}}}(s, t)=-b^{-1} D^{\prime}(s-t)$ and $C_{\widehat{\mathbf{I}}}(s, t)=D(s-t)$ is then a solution of (6.2)-(6.3) (hence its unique solution). Indeed, recall that $D(0)=1, D^{\prime}(0)=-b$ so the given initial conditions for $\left(R_{\widehat{\mathbf{I}}}, C_{\widehat{\mathbf{I}}}\right)$ hold. Further, these settings result with $\mu:=\rho+\widehat{\mathbf{I}}=\phi(1)$, so upon taking $u=t+\theta$ and $s=t+\tau$ we see that the equation (6.2), which is a direct consequence of (5.1) of Proposition 5.1, clearly holds. By the same transformations we see that our proposed solution satisfies (6.3) provided that

$$
D^{\prime}(\tau)=-\phi(1) D(\tau)-\int_{0}^{\tau} D(\tau-\theta) D^{\prime}(\theta) \phi^{\prime}(D(\theta)) d \theta+\phi(D(\tau))-b .
$$

Noting that by integration by parts,

$$
\int_{0}^{\tau} D(\tau-\theta) D^{\prime}(\theta) \phi^{\prime}(D(\theta)) d \theta=-\left.D(\tau-\theta) \phi(D(\theta))\right|_{0} ^{\tau}+\int_{0}^{\tau} D^{\prime}(\tau-\theta) \phi(D(\theta)) d \theta,
$$

and having $D(0)=1$, we see that (6.7) is equivalent to (1.20) hence holds as well.

Fixing $T<\infty$, for each $\xi$ positive let $\boldsymbol{\Gamma}_{\xi}:=\{(s, t): \xi \leq t \leq s \leq t+T\} \subset \mathbb{R}_{+} \times \mathbb{R}_{+}$. We proceed by showing that per fixed $\xi \geq 0$ and $T<\infty$ the mapping $I \mapsto\left(R_{I}, C_{I}\right)$ is Lipschitz with respect to the supremum norm over $\boldsymbol{\Gamma}_{\xi}$ with a Lipschitz constant that is independent of $\xi$. More precisely, given two bounded functions $I(s, t)$ and $\bar{I}(s, t)$ to which correspond continuously differentiable solutions $\left(R_{I}, C_{I}\right)$ and $\left(R_{\bar{I}}, C_{\bar{I}}\right)$ of $(6.2)-(6.3)$, such that $C_{I}$ and $C_{\bar{I}}$ are uniformly bounded, we let $\Delta_{\xi} I:=\sup \left\{|I(s, t)-\bar{I}(s, t)|:(s, t) \in \boldsymbol{\Gamma}_{\xi}\right\}$, and show that

$$
\begin{aligned}
& \Delta_{\xi} R:=\sup \left\{\left|R_{I}(s, t)-R_{\bar{I}}(s, t)\right|:(s, t) \in \boldsymbol{\Gamma}_{\xi}\right\} \leq \kappa_{0} \Delta_{\xi} I, \\
& \Delta_{\xi} C:=\sup \left\{\left|C_{I}(s, t)-C_{\bar{I}}(s, t)\right|:(s, t) \in \boldsymbol{\Gamma}_{\xi}\right\} \leq \kappa_{0} \Delta_{\xi} I,
\end{aligned}
$$


where $\kappa_{0}$ is a finite constant that depends only on $T, \beta, \nu^{\prime}, \rho$ and the uniform bound on $I, \bar{I}, C_{I}$ and $C_{\bar{I}}$ for $(s, t) \in \boldsymbol{\Gamma}_{\xi}$. Considering the bounded $\bar{I}=\widehat{\mathbf{I}}$, we have seen already that $R_{\bar{I}}(t+\tau, t)=R_{\mathrm{fdt}}(\tau)$ and $C_{\bar{I}}(t+\tau, t)=C_{\mathrm{fdt}}(\tau)$ with $C_{\bar{I}}$ bounded. Our assumption (6.5) then amounts to $\Delta_{\xi} I \rightarrow 0$ as $\xi \rightarrow \infty$. In particular, this implies that $I$ is also uniformly bounded on $\boldsymbol{\Gamma}_{\xi}$, so from (6.8) and (6.9) we get that $\Delta_{\xi} R \rightarrow 0$ and $\Delta_{\xi} C \rightarrow 0$, which are easily seen to match our desired conclusion.

We thus complete the proof by verifying the bounds of (6.8) and (6.9), by an argument similar to the one we used for proving uniqueness of the system of equations (1.14)-(1.16). To this end, note first that $R_{I}=\Lambda_{I} H_{C_{I}}$ for $\Lambda_{I}(s, t)=\exp \left(-\int_{t}^{s}(\rho+I(u, u)) d u\right)$ and $H_{C}(s, t)$ of $(2.2)$. Thus the assumed uniform bounds for $I$ and $C_{I}$ on $\boldsymbol{\Gamma}_{\xi}$ imply that $R_{I}$ is also uniformly bounded on $\boldsymbol{\Gamma}_{\xi}$ by a constant that depends only on $T, \beta, \rho, \nu^{\prime \prime}$ and the corresponding uniform bounds for $I, C_{I}$. Of course, the same applies for $R_{\bar{I}}$. Next let $\Delta I(v, u)=|I(v, u)-\bar{I}(v, u)|, \Delta R(v, u)=\left|R_{I}(v, u)-R_{\bar{I}}(v, u)\right|, \Delta C(v, u)=\left|C_{I}(v, u)-C_{\bar{I}}(v, u)\right|$ and for $(s, t) \in \boldsymbol{\Gamma}_{\xi}$ set

$$
h(s, t)=\int_{t}^{s}[\Delta R(s, u)+\Delta C(s, u)] d u .
$$

Then, similarly to the derivation of (2.7) and (2.8), upon considering the difference between the integrated form of (6.2)-(6.3) for our solutions $\left(C_{I}, R_{I}\right)$ and $\left(C_{\bar{I}}, R_{\bar{I}}\right)$, we find that for any $(s, t) \in \boldsymbol{\Gamma}_{\xi}$

$$
\begin{aligned}
\Delta R(s, t) & \leq \kappa_{1}\left[\int_{t}^{s} \Delta R(v, t) d v+\int_{t}^{s} h(v, t) d v+\int_{t}^{s} \Delta I(v, v) d v\right] \\
\Delta C(s, t) & \leq \kappa_{1}\left[\int_{t}^{s} \Delta C(v, t) d v+\int_{t}^{s} h(v, t) d v+\int_{t}^{s} \Delta I(v, t) d v+\int_{t}^{s} \Delta I(v, v) d v\right],
\end{aligned}
$$

where the positive $\kappa_{1}<\infty$ depends only on $T, \beta, \rho, \nu^{\prime}(\cdot)$ and the maximum of $I,\left|R_{I}\right|,\left|C_{I}\right|,|\bar{I}|,\left|R_{\bar{I}}\right|$ and $\left|C_{\bar{I}}\right|$ on $\boldsymbol{\Gamma}_{\xi}$. Replacing $t$ by $u$, summing these two inequalities and then integrating the result over $u \in[t, s]$ yields that

$$
h(s, t) \leq \kappa_{2}\left[\int_{t}^{s} h(v, t) d t+\int_{t}^{s} \Delta I(v, v) d v+\int_{t}^{s} \int_{t}^{v} \Delta I(v, u) d u d v\right]
$$

for any $(s, t) \in \boldsymbol{\Gamma}_{\xi}$, with a finite, positive constant $\kappa_{2}$ (of the same type of dependence as $\kappa_{1}$ ). Since $h(t, t)=0$ for all $t$, we get from (6.12) by Gronwall's lemma that $h(s, t) \leq \kappa_{3} \Delta_{\xi} I$ for some finite, positive $\kappa_{3}$ (of same dependence type as $\left.\kappa_{1}\right)$ and all $(s, t) \in \boldsymbol{\Gamma}_{\xi}$. Recall that $\Delta R(t, t)=\Delta C(t, t)=0$ due to the given initial conditions, so upon plugging into (6.10) and (6.11) our uniform bound on $h(s, t)$, we complete the proof of (6.8)-(6.9) by yet another application of Gronwall's lemma.

\section{REFERENCES}

[1] BEn AROUS, G. ; GUIOnNET, A. ; Large deviations for Langevin spin glass dynamics. Prob. Th. Rel. Fields 102, 455-509 (1995).

[2] BEN AROUS, G. ; GUIONNET, A. ; Symmetric Langevin spin glass dynamics. Ann. Probab. 25, 1367-1422 (1997).

[3] BEN AROUS, G. ; DEMBO, A. ; GUIONNET, A. Aging of spherical spin glasses. Probab. Theory Relat. Fields, 120:1-67 (2001).

[4] BEN AROUS, G. ; Aging and spin-glass dynamics. Proceedings of the International Congress of Mathematicians, Vol. III , 3-14, Higher Ed. Press, Beijing, 2002 (2002).

[5] BEN AROUS, G. ; DEMBO, A. ; GUIONNET, A. ; Cugliandolo-Kurchan equations for dynamics of Spin-Glasses. Submitted (2004).

[6] BOUCHEAUD J. ; CUGLIANDOLO L. ; KURCHAN J. ; MEZARD M. ; Mode coupling approximations, glass theory and disordered systems. Physica A, 226, 243-273. (1997)

[7] BOUCHAUD, J.P. ; CUGLIANDOLO, L.F. ; KURCHAN, J. ; MEZARD, M. ; Out of equilibrium dynamics in spin-glasses and other glassy systems. Spin glass dynamics and Random Fields, A. P Young Editor (1997).

[8] CUGLIANDOLO, L.F. ; Dynamics of glassy systems Les Houches (2002)

[9] CUGLiAnDOLO, L.F. ; DEAN, D.S. ; Full dynamical solution for a spherical spin-glass model. J. Phys. A: Math. Gen. 28, 4213-4234 (1995). 
[10] CUGLiAndolo, L.F. ; KURCHAN, J. ; Analytical solution of the off-equilibrium Dynamics of a Long-Range Spin-Glass Model.Phys. Rev. Lett. 71, 173 (1993)

[11] CUGLIANDOLO, L.F. ; LE DOUSSAL P.; Large time nonequilibrium dynamics of a particle in a random potential. Phys. Rev. E, 53, 1525-1552. (1996).

[12] GUIONNET, A. ; MAZZA, C.; Long time behaviour of non-commutative processes solution of a linear differential equation. Prob. Theory. Rel. Fields 131:493-518 (2005).

[13] GUIONNET, A.; Dynamics for spherical models of sping glass and Aging. Proceedings of the Ascona meeting 2004.

[14] GOTZE W. ; Liquids, Freezing and the Glass Transition Ed. J.P. Hansen, D. Levesque and J Zinn-Justin, Les Houches 1989, North-Holland. (1991)

[15] GOTZE W. ; SJOGREN L.; Relaxation processes in supercooled liquids. Rep. Prog. Phys., 55, 241-376. (1992)

[16] KRAICHNAN R. The structure of isotropic turbulence at very high Reynolds numbers. J. Fluid. Mech., 5, No. 4. $497-543$. (1959).

[17] KRAICHNAN R. Dynamics of nonlinear stochastic systems. J. Math. Phys., 2, No. 1. 124-148. (1961)

[18] McCOMB W. The Physics of Fluid Turbulence. Oxford University Press. (1990).

[19] MONIN A.; YAGLOM A.; Statistical Fluids Mechanics. Vol. 2. MIT Press, 1975.

[20] TALAGRAND, M. Free energy of the spherical mean field model. To appear in Prob. Th. Rel. Fields (2007).

Department of Statistics and Department of Mathematics, Stanford University, Stanford, CA 94305.

E-mail address: amir@math.stanford.edu

UMPA, Ecole Normale Superieure de Lyon, 46 allée d'Italie, 69364 Lyon Cedex 07, France

E-mail address: Alice.Guionnet@umpa.ens-lyon.fr

Département de mathématiques, Université Pérolles, Chemin du Musée 23, CH-1700 Fribourg, Suisse 